\title{
Equilibrium price with institutional investors and with naive traders *
}

\author{
Dominique Dupont \\ Division of Monetary Affairs \\ Board of Governors of the Federal Reserve System
}

April 1998

${ }^{*}$ Mail Stop 69, Federal Reserve Board, Washington, DC 20551; phone: (202) 452-6483; fax: (202) 452-2301; e-mail: m1dyd99@frb.gov. I thank Vincent Reinhart and the participants of the R\&S lunch-time workshop for helpful discussions. The views expressed herein are the author's and do not necessarily reflect those of the Board of Governors or the Federal Reserve System.

JEL Classification: G14, Financial Economics, Information and Market Efficiency 


\begin{abstract}
This paper uses an equilibrium model to study how institutional investors influence the volatility and the informativeness of asset prices. The growth in the proportion of U.S. equities owned by institutions in the past two decades, their resulting dominant position in financial markets, and empirical evidence that institutional ownership may increase volatility warrant studying this issue from a theoretical standpoint. In this paper, institutional investors are assumed to be "rational" informed traders while individual investors are supposed to be "naive" informed traders, insofar as the former use the equilibrium price to extract information while the latter do not. Using a framework with a competitive market, multiple informed traders and one liquidity trader, the paper compares the informativeness and the volatility of the equilibrium price in an economy where the informed traders are naive and in one where they are rational. The model also studies how the informativeness and the volatility of the price react to changes in parameters such as the quality of the information of the informed traders, their aversion to risk, the variance of the true asset price, etc. The paper finally investigates how an increase in the number of informed traders, whether they are rational or naive, affects the price variance, and assesses the impact of transaction costs on the variance of the price and its informativeness.
\end{abstract}




\section{Introduction}

Do institutional investors, presumably sophisticated ones, make asset prices less volatile and more informative or the reverse? The growing role of institutional investors in the past decade warrants more efforts to answer this question. The relationship between institutional ownership and volatility has been investigated empirically by Sias (1996) and broad implications of ownership patterns on financial markets has been discussed in Friedman (1996). The objective of this paper is to analyze the effect of institutional investors on the market price by using a theoretical framework and assuming those investors are more sophisticated than individual investors, insofar as the former use the equilibrium price to extract information while the latter do not.

Using the securities listed on the New York Stock Exchange between 1977 and 1991, and controlling for capitalization, Sias (1996) finds a positive relationship between the fraction of shares held by institutional investors and the volatility of the stock returns ${ }^{1}$ and presents evidence that the increase in the share of institutional ownership precedes the increase in volatility, not the reverse. Sias also points to the apparent inconsistency between this evidence and most of the extant literature, from which he quotes three arguments implying a negative relationship between volatility and institutional ownership. First, fund managers may shy away from riskier stocks for prudential reasons (e.g. to prevent lawsuits). Second, institutional investors, being able to invest more in research, have more and better information about the companies in their portfolio. Third, institutional investors should be more likely to behave rationally. ${ }^{2}$ In this paper, we argue that it is precisely the institutional investors' ability to rationally use all the available information-including the market price - that induces a higher price volatility (and a lower price informativeness) in the stocks they dominate. Friedman (1996) discusses a series of implications of the growing weight of institutional ownership on the proper workings of the financial markets. He notes that the increasing concentration of decision-making could render the market price more sensitive to each investor's idiosyncratic shocks, which would translate into increased volatility. My model investigates whether an increase in the number of institutional investors offsets this effect.

The relationship between institutional ownership and the volatility and the informativeness of

\footnotetext{
${ }^{1}$ Without controlling for capitalization, institutional investors' ownership and stock return volatility are negatively correlated because institutional investors tend to hold stocks with large capitalization and the latter tend to be less volatile.

${ }^{2}$ Sias also cites some arguments that could lead to the opposite conclusion, like the asymmetry in incentives for the fund manager.
} 
asset prices is an important issue, especially since institutional investors are playing a growing role in financial markets. The share of corporate equities held by U.S. institutions has jumped from about 27.3 percent in 1970 to above 40 percent in the 1990s, reaching about 47 percent in the third quarter of 1997. ${ }^{3}$ The growing importance of institutional investors is also witnessed by the triennial Survey of Consumer Finances sponsored by the Federal Reserve Board. The share of the households' financial wealth owned indirectly — in the form of mutual funds, pension plans, life insurance or other managed accounts - rose from about 37 percent in 1989 to about 52 percent in 1995. ${ }^{4}$ There is no evidence that the growth of the share of financial assets held by institutional investors should taper off significantly in the near future. On the contrary, the current uncertainties about the future of the Social Security program might prompt more Americans to step up their investments in mutual funds and retirement accounts, reinforcing the role of institutional investors.

We study the effect of institutional investors' ownership on asset prices by using a static model where informed and liquidity traders act competitively, and assuming that the informed traders are alternatively "rational" or "naive" 5 . Institutional investors are more able to observe current market conditions and act rapidly than individual stock owners. Therefore, it is natural to model the former as rational and the latter as naive. The informed traders always observe a private signal correlated with the true value of the asset, but rational informed traders also condition on the market-clearing price when they decide how much of the traded asset they want to hold, whereas naive informed traders condition only on their signals. The liquidity trader is neither rational nor naive but is price sensitive.

The assumption that informed traders are rational or that they are naive can lead to very different conclusions. A well known example is provided by the no-trade theorems. To summarize, if the motivation to trade is not the rebalancing of portfolios but private information and if there is no exogenous noise trading, rational agents will gather that anyone accepting to take the counterpart of their trades would only do so because he or she possesses an informational advantage, and hence that the initiating party would suffer a loss. Consequently, in equilibrium, no trade takes place. 6 In our model too, the absence of noise precludes trade. In that case, the equilibrium price

\footnotetext{
${ }^{3}$ Source: 1996 NYSE Fact Book and Federal Reserve Board, "Flow of funds".

${ }^{4}$ Source: Federal Reserve Bulletin, January 1997, p. 6, Table 4.

${ }^{5}$ For the sake of simplicity, we assume that the informed traders are all naive or all rational. If naive and rational traders exist simultaneously, the model cannot be solved in closed form.

${ }^{6}$ The no-trade theorems do not exactly have the same implications. For example, Milgrom and Stockey (1982)
} 
reveals all the information, that is, equals the conditional expectation of the true value of the asset conditioned on all the informed traders' signals.

The first section presents the model in the general case and when the informed traders have equally precise signals (this assumption will be maintained in the rest of the paper). The objective throughout is to compare the equilibria when the informed traders are rational and when they are naive, and to study the variance and the informativeness (defined as the square of the correlation between the equilibrium price and the true value of the asset). The second section specializes the model to two informed traders and one liquidity trader, allowing the two informed traders' signals to be correlated. The third section generalizes the analysis to $n$ informed traders but restricts their signals to be independent. It also investigates the effect of introducing a transaction cost (or tax) and studies the expected trading volume. The fourth section introduces positive correlation across the informed traders' signals by using a "measurement-error" approach where each private signal is the sum of the true asset value, a systematic, and an idiosyncratic noise. Unlike in the previous section, this framework allows the number of informed traders to grow without bounds. We can hence study the limit and the convergence properties of the market price as the number of traders becomes infinite when traders are naive and when they are rational. In the following, institutional investors are called rational and the individual investors naive. However, the latter are not irrational noise traders.

\section{Model}

The paper uses a static, competitive, equilibrium framework with one liquidity trader and $n$ informed traders, each observing a signal $G_{i}, i=1, \ldots, n$, correlated with the true value of the asset. For each index $i$, the random variable $G_{i}$ has mean zero and variance one, the vector $\left(G_{1}, \ldots, G_{n}, x\right)$ is normally distributed. Each trader has a CARA utility function $u_{i}\left(\pi_{i}\right)$ with common risk aversion coefficient $\lambda$. Agent $i$ 's profit, $\pi_{i}$, is the random variable $y_{i}(x-p)$, where $y_{i}$ is the demand of trader $i, x$ the true value of the asset, and $p$ the market price. The $(n+1)^{t h}$ trader is a noise trader, whose demand is $y_{n+1}=-s(p-\varepsilon)$, where $s>0$, and $\varepsilon$ is uncorrelated with the true value of the asset and the informed traders' signals. The framework described above must be seen as a 'reduced form' of a structural model, which would introduce another period where agents consume but do not trade, and a riskless asset that traders could purchase or sell to finance to desired holdings of

implies that agents are indifferent to trading, not that they won't trade. 
risky securities, as in Diamond and Verrechia (1981).

When the informed traders are rational, their valuations are $v_{i}=E\left[x \mid G_{i}, p\right]$; when they are naive, their valuations are $v_{i}=E\left[x \mid G_{i}\right]$. As $\left(p, G_{1}, \ldots, G_{n}\right)$ is normally distributed, informed trader $i$ 's demand is $y_{i}(p)=\alpha_{i}\left(v_{i}-p\right)$, with $\alpha_{i}=\frac{1}{\lambda v a r\left[x \mid G_{i}, p\right]}$ when informed traders are rational and $\alpha_{i}=\frac{1}{\lambda v a r\left[x \mid G_{i}\right]}$ when they are naive. In all cases, $p=\Sigma_{1}^{n} \theta_{i} v_{i}+\theta_{n+1} \varepsilon$, with $\alpha=\Sigma_{1}^{n} \alpha_{i}, \theta_{i}=\frac{\alpha_{i}}{s+\alpha}$, for $i=1, \ldots, n$, and $\theta_{n+1}=\frac{s}{s+\alpha}$. Note that $\Sigma_{i=1}^{n+1} \theta_{i}=1, \theta_{i}$ is the weight of the $i^{\text {th }}$ trader in the determination of the market-clearing price. As a result, rational trader $i$ 's equilibrium valuation is $v_{i}$ such that

$$
v_{i}=E\left[x \mid G_{i}, \Sigma_{j=1}^{n} \theta_{j} v_{j}+\theta_{n+1} \varepsilon\right]
$$

Equation (1) is an example of 'infinite regress'. For any two agents $i$ and $j$, agent $i$ 's valuation depends on agent $j$ 's valuation, in turn, agent $j$ 's valuation depends on agent $i$ 's valuation, and so forth. Besides, in (1), traders have to take into account the 'noise' injected in the trading by $\varepsilon$. Equilibrium valuations can be thought of as solutions to $n$ simultaneous equations similar to (1), with $i=1, \ldots, n$. As proposition (1) shows, if there is a normally distributed solution $v^{*}$ to (1), then the equilibrium price is linear in the traders signals and the liquidity noise.

Proposition 1 If there is a vector $v^{*}$ satisfying (1) and $v^{*}, G, x$ and $\varepsilon$ are jointly normally distributed, the corresponding equilibrium price $p^{*}$ is linear in the informed traders' signals and the noise trader's liquidity shock, that is,

$$
p^{*}=\sum_{i=1}^{n} a_{i} G_{i}+a_{n+1} \varepsilon
$$

Proof of proposition 1: Suppose there is a normally distributed fixed point $z^{*}=\left(v^{*}, G, \varepsilon, x\right)$. Recall that $p^{*}=\Sigma_{i=1}^{n} \theta_{i}^{*} v_{i}^{*}+\theta_{n+1}^{*} \varepsilon$. Consequently, $z_{i}^{*}=\left(G_{i}, p^{*}\right)$ is a normally distributed vector, and $v_{i}^{*}=E\left[x \mid G_{i}, p^{*}\right]$ is linear in $G_{i}$ and $p^{*}$. Writing $v_{i}^{*}=c_{i} G_{i}+d_{i} p^{*}$, we get that $p^{*}$ verifies the following equation

$$
\left(1-\sum_{i=1}^{n} \theta_{i} d_{i}\right) p^{*}=\Sigma_{i=1}^{n} \theta_{i}^{*} c_{i} G_{i}+\theta_{n+1}^{*} \varepsilon
$$

Suppose that $\sum_{i=1}^{n} \theta_{i}^{*} d_{i}=1$, then $\varepsilon$ and the $G_{i}$ would be correlated, which contradicts the assumption that the liquidity shock is uncorrelated with the signals. Hence, $\Sigma_{i=1}^{n} \theta_{i}^{*} d_{i} \neq 1$, and $p^{*}$ can be written as a linear combination of the state variables. When the signals are identically distributed, the $i$ indexes can be permuted and consequently, we can write $a_{i}=a$ for $i=1, \ldots, n$ and $a_{n+1}=b$. Naturally, equation (2) also holds when the informed traders are naive. 
The objective of the paper is to characterize the equilibrium price when the informed traders are rational and when they are naive, that is, when they take into account the market-clearing price in their conditioning information and when they don't. We impose that the informed traders' signals be identically distributed in order to get a closed-form solution for the equilibrium price. We focus on how the variance of the price and its informativeness - defined as the ratio of the variance of $E[x \mid p]$ to that of $x$ or, equivalently, as the square of the correlation between $x$ and $p$-respond to changes in parameters, such as the risk aversion of the informed traders, the precision of their signals, and the variance of the liquidity shock. The price variance is noted $v a r_{p}^{N}$ when traders are naive, and $\operatorname{var}_{p}^{R}$ when traders are rational; the price informativeness is noted $I_{p}^{N}$ when traders are naive, $I_{p}^{R}$ when traders are rational. First, we limit the number of informed traders to two, but we allow for correlation between their signals. Then, we allow for $n$ informed traders, but we suppose their signals are uncorrelated.

The market-clearing price, $p$, is an average of the informed traders' valuations, $v_{i}$, and the noise, $\varepsilon$. Assuming the informed traders' signals $G_{i}$ are identically distributed, the pricing equation simplifies to

$$
p=\theta \sum_{i=1}^{n} v_{i}+(1-n \theta) \varepsilon
$$

where $v_{i}=E\left[x \mid G_{i}\right]$ when traders are naive and $v_{i}=E\left[x \mid G_{i}, p\right]$ when they are rational, $\theta=$ $\frac{1}{n+\kappa\left(\sigma_{x}^{2}-E\left[v_{i}^{2}\right]\right)}$, where $\kappa=s \lambda . \kappa$ is the ratio of the slope of the liquidity demand to the coefficient of risk tolerance and measures the relative power of the liquidity trader and the informed traders in determining the equilibrium price. The more strongly the liquidity trader reacts to price changes, or the more risk averse the informed trader is, the greater the influence of the liquidity trader in determining the equilibrium price and the higher $\kappa$. From proposition (1), and since the $G_{i}$ are identically distributed, the equilibrium price is also such that

$$
p=a \sum_{i=1}^{n} G_{i}+b \varepsilon
$$

The key is to find values for $a$ and $b$ so that equations (4) and (5) simultaneously hold. This will be achieved by first solving for the equilibrium value of the ratio $\frac{b}{a}$, which we call $\beta$. 


\section{Equilibrium with two informed traders.}

Let $\rho$ be the common value of the correlation between each informed trader's signal and the true value of the asset and $\gamma$ be the correlation between the two informed traders' signals. The coefficients $\rho$ and $\gamma$ measure, respectively, how well informed each informed trader is, and how much the two informed traders agree with one another. The informativeness and the variance of the price are, respectively:

- $I_{p}=\frac{(2 \rho)^{2}}{2(1+\gamma)+\sigma_{\varepsilon}^{2} \beta^{2}}$,

- $\operatorname{var}_{p}=2(1+\gamma) a^{2}+\sigma_{\varepsilon}^{2} b^{2}$.

In the following, the coefficients of the private signals and of the liquidity shock in the pricing equation are denoted respectively by $a^{N}$ and $b^{N}$ when traders are naive, and by $a^{R}$ and $b^{R}$ when traders are rational. The ratio of the coefficient of the liquidity shock to that of the private signals is noted $\beta^{N}$ when traders are naive and $\beta^{R}$ when they are rational. First, we compare the volatility and the informativeness of the equilibrium price when the traders are naive and when they are rational. Then, we study how these two variables react to changes in the parameters.

Proposition 2 The equilibrium price is more volatile and less informative when traders are rational than when they are naive. This is because $a^{R}>a^{N}, b^{R}>b^{N}$, and $\beta^{R}>\beta^{N}$.

The proof of proposition (2) is presented further below. Many results linking the informativeness and the volatility of the price are derived analytically. For some, though, the complexity of the closed functional forms called for the use of numerical examples.

Proposition 3 Both when informed traders are rational and when they are naive, the informativeness of the equilibrium price is increasing in $\rho$ and decreasing in $\kappa, \sigma_{x}$ and $\sigma_{\varepsilon}$. The price informativeness decreases in $\gamma$ for all values of $\gamma$ assuming the traders are naive and for $\gamma \leq \rho^{2}$ assuming they are rational.

The proof of proposition (3) are presented further below. Moreover, numerical examples show that, when traders are rational, the price informativeness can be increasing in $\gamma$ for $\gamma>\rho^{2}$. As for the price variance, while it can be proven that it is increasing in $\gamma$ and $\sigma_{\varepsilon}$ when traders are naive, we had to resort to numerical examples to investigate other parameters or when assuming that traders were rational. The following tries to shed some intuition on the results of proposition (3) and those about the price variance. 
As expected, the better informed the traders are (the higher $\rho$ ), the more informative is the equilibrium price. Also, quite naturally, an increase in the volatility of the true asset value, in the risk aversion of the informed traders, or in the liquidity traders' reactiveness to price depresses the informativeness of price. When the volatility of the true asset value picks up or informed traders become more risk averse, those trade less aggressively on their private signals, transmitting less information into the price. As a consequence, whether traders are rational or naive, increasing $\kappa$ reduces the informativeness of the price. Numerical examples show that raising $\kappa$ can also reduce the price variance.

Intuitively, when the traders' signals become less correlated (lower $\gamma$ ), the equilibrium price should contain more information: since the price incorporates the traders' signals, the less overlap in the information of each trader, the more information should be available overall for the price to reflect. Moreover, one would expect an increase in $\gamma$ to make the equilibrium price more volatile because the price is an average of the informed traders' signals and the noise and, when $\gamma$ increases, the realizations of the informed traders' signals are more likely to be of the same magnitude. This intuition is entirely correct when traders are naive because the coefficient $a^{N}$ and $b^{N}$ in the pricing equation do not depend on $\gamma$. However, when traders are rational, the coefficients $a^{R}$ and $b^{R}$ depend on $\gamma$. When traders are rational, each of them takes account of the correlation of his signal with the other trader's signal when deciding how much to trade. Numerical examples indicate that $a^{R}$ and $b^{R}$ are decreasing in $\gamma$, which, on net, can make $v a r_{p}^{R}$ a decreasing function of $\gamma$. Also, the price informativeness can be increasing in $\gamma$ for $\gamma>\rho^{2}$.

Both when traders are naive and when they are rational, an increase in the volatility of the liquidity shock, $\sigma_{\varepsilon}$, reduces the informativeness of the price. But the effect on the price variance will differ. Because, when traders are naive, $a^{N}$ and $b^{N}$ are independent of $\sigma_{\varepsilon}$, the equilibrium price variance, $\operatorname{var}_{p}^{N}$, is increasing in $\sigma_{\varepsilon}$. However, when traders are rational, the intensity of the noise - measured by its variance - will bear on how much information they reveal in equilibrium. Although it is proven that, on net, the informativeness of price is still decreasing in $\sigma_{\varepsilon}$, the effect on its variance is not as simple. Numerical examples show that, as $\sigma_{\varepsilon}$ increases, the price variance decreases at first, and then increases. 


\subsection{Equilibrium with naive traders}

The equilibrium price when informed traders are naive is $p=\theta\left(v_{1}+v_{2}\right)+(1-2 \theta) \varepsilon$, with $\theta^{-1}=2+\kappa \sigma_{x}^{2}\left(1-\rho^{2}\right)$. Consequently,

$$
\begin{aligned}
a^{N} & =\frac{\sigma_{x} \rho}{2+\kappa \sigma_{x}^{2}\left(1-\rho^{2}\right)}, \\
b^{N} & =\frac{\kappa \sigma_{x}^{2}\left(1-\rho^{2}\right)}{2+\kappa \sigma_{x}^{2}\left(1-\rho^{2}\right)}, \\
\beta^{N} & =\kappa \sigma_{x} \frac{\left(1-\rho^{2}\right)}{\rho} .
\end{aligned}
$$

$\beta^{N}$ is increasing in $\sigma_{x}, \kappa$, decreasing in $\rho$, and independent of $\gamma$ and $\sigma_{\varepsilon}$.

\subsection{Equilibrium with rational traders.}

In the rational case, trader 1's equilibrium valuation is $v_{1}$ such that,

$$
\begin{aligned}
v_{1} & =E\left[x \mid G_{1}, p\right] \\
& =E\left[x \mid G_{1}, a\left(G_{1}+G_{2}\right)+b \varepsilon\right] \\
& =E\left[x \mid G_{1}, G_{2}+\beta \varepsilon\right] \\
& =E\left[x \mid G_{1}, G_{2}-\gamma G_{1}+\beta \varepsilon\right] \\
& =E\left[x \mid G_{1}\right]+E\left[x \mid G_{2}-\gamma G_{1}+\beta \varepsilon\right] \\
& =\operatorname{cov}\left(x, G_{1}\right) G_{1}+\frac{\operatorname{cov}\left(x, G_{2}-\gamma G_{1}+\beta \varepsilon\right)}{\operatorname{var}\left(G_{2}-\gamma G_{1}+\beta \varepsilon\right)}\left(G_{2}-\gamma G_{1}+\beta \varepsilon\right) \\
& =\rho \sigma_{x} G_{1}+\frac{\rho \sigma_{x}(1-\gamma)}{1-\gamma^{2}+\beta^{2} \sigma_{\varepsilon}^{2}}\left(G_{2}-\gamma G_{1}+\beta \varepsilon\right)
\end{aligned}
$$

The fifth line comes from the fact that $G_{2}-\gamma G_{1}$ and $\varepsilon$ are uncorrelated with $G_{1}$ and that all the variables are jointly normally distributed. We conclude that

$$
\begin{aligned}
v_{1}+v_{2} & =\rho \sigma_{x}\left(G_{1}+G_{2}\right)+\frac{\rho \sigma_{x}(1-\gamma)^{2}}{1-\gamma^{2}+\beta^{2} \sigma_{\varepsilon}^{2}}\left(G_{1}+G_{2}\right)+\frac{2 \rho \sigma_{x}(1-\gamma) \beta}{1-\gamma^{2}+\beta^{2} \sigma_{\varepsilon}^{2}} \varepsilon \\
& =\rho \sigma_{x} \frac{2(1-\gamma)+\beta^{2} \sigma_{\varepsilon}^{2}}{1-\gamma^{2}+\beta^{2} \sigma_{\varepsilon}^{2}}\left(G_{1}+G_{2}\right)+\rho \sigma_{x} \frac{2(1-\gamma) \beta}{1-\gamma^{2}+\beta^{2} \sigma_{\varepsilon}^{2}} \varepsilon
\end{aligned}
$$

Combining equations (5) and (8), and using the fact that $\theta^{-1}-2=\kappa \sigma_{x}^{2}\left(1-\rho^{2} \frac{2(1-\gamma)+\beta^{2} \sigma_{\varepsilon}^{2}}{1-\gamma^{2}+\beta^{2} \sigma_{\varepsilon}^{2}}\right)$, we get

$$
\begin{aligned}
\theta^{-1}\left(1-\gamma^{2}+\beta^{2} \sigma_{\varepsilon}^{2}\right) p & =\rho \sigma_{x}\left(2(1-\gamma)+\beta^{2} \sigma_{\varepsilon}^{2}\right)\left(G_{1}+G_{2}\right) \\
& +\left\{\kappa \sigma_{x}^{2}\left[1-\gamma^{2}-2(1-\gamma) \rho^{2}+\left(1-\rho^{2}\right) \beta^{2} \sigma_{\varepsilon}^{2}\right]+2 \rho \sigma_{x}(1-\gamma) \beta\right\} \varepsilon
\end{aligned}
$$


The equilibrium $\beta$ must be such that

$$
\beta=\frac{\kappa \sigma_{x}\left[(1-\gamma)\left(1+\gamma-2 \rho^{2}\right)+\left(1-\rho^{2}\right) \beta^{2} \sigma_{\varepsilon}^{2}\right]+2 \rho(1-\gamma) \beta}{\rho\left(2(1-\gamma)+\beta^{2} \sigma_{\varepsilon}^{2}\right)}
$$

The equilibrium $\beta$ must solve the following cubic equation.

$$
\varphi\left(\rho, \gamma, \kappa \sigma_{x}, \sigma_{\varepsilon}, \beta\right)=\rho \sigma_{\varepsilon}^{2} \beta^{3}-\kappa \sigma_{x}\left(1-\rho^{2}\right) \sigma_{\varepsilon}^{2} \beta^{2}+\kappa \sigma_{x}(1-\gamma)\left(2 \rho^{2}-\gamma-1\right)=0
$$

Likewise, the equilibrium $\beta$ is the solution of the equation

$$
u\left(\beta, \rho, \kappa \sigma_{x}\right)=v\left(\beta, \gamma, \sigma_{\varepsilon}^{2}, \rho, \kappa \sigma_{x}\right),
$$

with

$$
\begin{aligned}
& u\left(\beta, \rho, \kappa \sigma_{x}\right)=\rho \beta^{3}-\kappa \sigma_{x}\left(1-\rho^{2}\right) \beta^{2} \\
& v\left(\gamma, \sigma_{\varepsilon}^{2}, \rho, \kappa \sigma_{x}\right)=\frac{\kappa \sigma_{x}}{\sigma_{\varepsilon}^{2}}(1-\gamma)\left(\gamma+1-2 \rho^{2}\right)
\end{aligned}
$$

The functions $u$ and $v$ are displayed on Figure (1). We impose $-1<\gamma<1,0<\rho<1$ and $\gamma-2 \rho^{2}+1>0$ so that the covariance matrix of state vector $\left(G_{1}, G_{2}, x, \varepsilon\right)$ is positive definite. ${ }^{7}$ The function $u$ is cubic in $\beta$, it is zero for $\beta=0$ and $\beta=\kappa \sigma_{x} \frac{1-\rho^{2}}{\rho}$, it is negative or zero on for $\beta \in\left(-\infty, \kappa \sigma_{x} \frac{1-\rho^{2}}{\rho}\right]$ and positive for $\beta \in\left(\kappa \sigma_{x} \frac{1-\rho^{2}}{\rho},+\infty\right)$. The function $v$ is quadratic in $\gamma$ and positive for the admissible $\gamma$ and $\rho$. As a result, there is a unique real solution to equation (12), call it $\beta^{R}\left(\gamma, \sigma_{\varepsilon}^{2}, \rho, \kappa \sigma_{x}\right)$. Since the function $v$ is positive, the solution to (12) must lay above $\kappa \sigma_{x} \frac{1-\rho^{2}}{\rho}$, which is the value of $\beta^{N}$. Hence $\beta^{R} \geq \beta^{N}$, and $\beta^{N}$ and $\beta^{R}$ coincide only for $\gamma=1$ (in which case the covariance matrix becomes singular).

Proposition $4 \beta^{R}$ is increasing in $\sigma_{x}, \kappa$, and decreasing in $\rho, \sigma_{\varepsilon}$, increasing in $\gamma$ for $\gamma \leq \rho^{2}$ and decreasing in $\gamma$ for $\gamma>\rho^{2}$. Furthermore, $a^{R}>a^{N}, b^{R}>b^{N}$ and $\beta^{R}>\beta^{N}$.

The latter implication makes the price more volatile and less informative when traders are rational than when they are naive. The results of proposition (4) are presented graphically first. In the following, one should remain aware that the solution to (12) is the value of $\beta$ right of $\kappa \sigma_{x} \frac{1-\rho^{2}}{\rho}$ where

${ }^{7}$ The matrix $\left(\begin{array}{llll}1 & \gamma & \rho & 0 \\ \gamma & 1 & \rho & 0 \\ \rho & \rho & 1 & 0 \\ 0 & 0 & 0 & \sigma_{\varepsilon}^{2}\end{array}\right)$ with $-1<\gamma<1$ and $0<\rho<1$ is the covariance matrix of $\left(G_{1}, G_{2}, x, \varepsilon\right)$ provided it is positive definite, i.e, if and only if $\gamma>2 \rho^{2}-1$. Once $\gamma$ has been chosen, $\rho$ must be between 0 and $\sqrt{\frac{\gamma+1}{2}}$. 
$v$ and $u$ intersect. Increasing $\sigma_{\varepsilon}$ shifts $v$ downwards, but does not affect $u$, therefore $\beta$ is a decreasing function of $\sigma_{\varepsilon}$. Increasing $\kappa$ or $\sigma_{x}$ pushes $v$ up and $u$ down, therefore $\beta$ is an increasing function of those parameters. Similarly, increasing $\rho$ shifts $u$ up and $v$ down, lowering the equilibrium $\beta$. Since increasing the value of $\gamma$ from its minimum value first shifts $v$ up and then down, and $u$ is independent of $\gamma, \beta$ is increasing in $\gamma$ for $\gamma \in\left(1-2 \rho^{2}, \rho^{2}\right]$ and decreasing on $\left(\rho^{2}, 1\right)$. The results of proposition (4) can be also introduced using the implied function theorem. Let $\mu=\kappa \sigma_{x}$ and $\beta=\phi\left(\rho, \gamma, \mu, \sigma_{\varepsilon}\right)$ the $\beta$ such that $\varphi\left(\rho, \gamma, \mu, \sigma_{\varepsilon}, \beta\right)=0$. Let $x \in\left\{\rho, \gamma, \mu, \sigma_{\varepsilon}\right\}$, then $\frac{\partial \phi}{\partial x}=-\frac{\frac{\partial \varphi}{\partial x}}{\frac{\partial \varphi}{\partial \beta}}$ for non-zero values of the denominator. $\frac{\partial \varphi}{\partial \beta}=\sigma_{\varepsilon}^{2} \beta\left(3 \rho \beta-2 \mu\left(1-\rho^{2}\right)\right)$ is positive since $\beta>\mu \frac{1-\rho^{2}}{\rho}$ for admissible values of $\gamma$ and $\rho$. Also, $\frac{\partial \varphi}{\partial \rho}>0, \frac{\partial \varphi}{\partial \mu}<0, \frac{\partial \varphi}{\partial \sigma_{\varepsilon}}>0$, and $\frac{\partial \varphi}{\partial \gamma}$ is negative for $\gamma<\rho^{2}$ and positive for $\gamma>\rho^{2}$. Hence, the equilibrium $\beta$ is decreasing in $\rho$ and $\sigma_{\varepsilon}$, increasing in $\kappa$ and $\sigma_{x}$, increasing in $\gamma$ for $\gamma \leq \rho^{2}$ and decreasing in $\gamma$ for $\gamma>\rho^{2}$.

The price informativeness is $I\left(\beta, \rho, \sigma_{\varepsilon}^{2}\right)=\frac{(2 \rho)^{2}}{2(1+\gamma)+\sigma_{\varepsilon}^{2} \beta^{2}}$, where $\beta$ is the equilibrium $\beta^{R}$ or $\beta^{N}$. It follows that the price is always less informative than with naive traders. The informativeness is equal across the two types of traders at the boundary of the parameter space, i.e., if $\gamma=1$ or $\gamma=2 \rho^{2}-1$, or if $\kappa \sigma_{x}=0$ (that is, if there is no noise traders or, at the limit, if the informed traders are risk-neutral). With both rational and naive traders, the price informativeness is decreasing in $\kappa \sigma_{x}$.

Recall that, when traders are rational, $a=\frac{\rho \sigma_{x}\left[2(1-\gamma)+\beta^{2} \sigma_{\varepsilon}^{2}\right]}{\theta^{-1}\left(1-\gamma^{2}+\beta^{2} \sigma_{\varepsilon}^{2}\right)}$. Substituting in $\theta^{-1}=2+$ $\left.\kappa \sigma_{x}^{2}\left(1-\rho^{2} \frac{2(1-\gamma)+\beta^{2} \sigma_{\varepsilon}^{2}}{1-\gamma^{2}+\beta^{2} \sigma_{\varepsilon}^{2}}\right)\right)$ one gets,

$$
a(\beta, \gamma)=\frac{\rho \sigma_{x} \beta^{2} \sigma_{\varepsilon}^{2}+2 \rho \sigma_{x}(1-\gamma)}{\left(2+\kappa \sigma_{x}^{2}\left(1-\rho^{2}\right)\right) \beta^{2} \sigma_{\varepsilon}^{2}+\left(2(1+\gamma)+\kappa \sigma_{x}^{2}\left(1+\gamma-2 \rho^{2}\right)\right)(1-\gamma)}
$$

$a(\beta, 1)=\frac{\rho \sigma_{x}}{2+\kappa \sigma_{x}^{2}\left(1-\rho^{2}\right)}=a^{N}$ does not depend on $\beta$. Besides, since $2 \rho^{2}-1<\gamma<1, \frac{\partial a}{\partial \gamma}<0$ : $a(\beta, \gamma)$ is a decreasing function of $\gamma$ and $a(\beta, 1)$ is independent of $\beta$. Hence, $a(\beta, \gamma)>a^{N}$ for all $\beta$. Consequently, for $2 \rho^{2}-1<\gamma<1, a^{R}>a^{N}$. Moreover, we showed that $\beta^{R}>\beta^{N}$. Hence, $b^{R}>b^{N}$, and, since $\operatorname{var}_{p}=2 a^{2}(2+\gamma)+\sigma_{\varepsilon}^{2} b^{2}$, and $I_{p}=\frac{(2 \rho)^{2}}{2(1+\gamma)+\sigma_{\varepsilon}^{2} \beta^{2}}$, the price variance is greater and the price informativeness lower with rational traders than with naive ones.

The noise variance affects $I_{p}$ through $\sigma_{\varepsilon} \beta$, which is increasing in $\sigma_{\varepsilon}$. This result can also be introduced using the implicit function theorem and noting that $\frac{\partial}{\partial \sigma_{\varepsilon}}=\left(\phi \sigma_{\varepsilon}\right)$ has the same sign as $\beta \frac{\partial \varphi}{\partial \beta}-\sigma_{\varepsilon} \frac{\partial \varphi}{\partial \sigma_{\varepsilon}}=\sigma_{\varepsilon}^{2} \rho \beta^{3}>0$ 


\section{Equilibrium with multiple informed traders with identically dis- tributed signals}

In that case, the informativeness of the price and its variance are, respectively:

- $I_{p}=\frac{\rho^{2} n^{2}}{n+\beta^{2} \sigma_{\varepsilon}^{2}}$

- $\operatorname{var}_{p}=a^{2} n+b^{2} \sigma_{\varepsilon}^{2}$.

Proposition 5 Both when informed traders are rational and when they are naive, the informativeness of the equilibrium price is increasing in $\rho$ and decreasing in $\kappa, \sigma_{x}$, and $\sigma_{\varepsilon}$. The price informativeness increases in $n$ for all values of $n$ when traders are naive, and for $n \geq \frac{1+\rho^{2}}{2 \rho^{2}}$ when they are rational.

Proofs of proposition (5) are presented in the following sections. When traders are naive, the price variance is decreasing in $n$ after some threshold. A very interesting result of the numerical examples is that, when the informed traders are rational, the price variance can be increasing in the number of traders (see Figure (3)). This result stands in contrast with the argument of Friedman (1996) who attributes the increased price volatility brought about by the growing role of institutional of investors to a reduction of the number of agents acting independently. Two effects are at play when more rational traders enter the market with identically and independently distributed private signals. First, since the price is an average of those signals, it tends to become less volatile. This is quite similar to the decrease in price volatility brought about by averaging a larger number of orders in Pagano (1989), although demands for the asset in that model stem from the rebalancing of portfolios, not from private information. When traders are naive, only this averaging effect exists. Moreover, with both naive and rational traders, an increase in the pool of informed traders brings more information into the market (see Figure (2)), because, even though their signals have the same distribution, they may have different realizations (all the more so as they are independently distributed). The increase in the available information, which is reflected by the price in equilibrium, affects differently the demand of naive traders and of rational traders. Loosely speaking, rational traders are aware that the price is more informative because they include it in their conditioning information. Consequently, they react more strongly to a deviation between their valuation, $v_{i}$, and the market price, $p$. In contrast, the naive traders don't. More precisely, the demand of each trader equals the product of $v_{i}-p$ and $\alpha_{i}=\frac{1}{\lambda \operatorname{var}\left(x \mid H_{i}\right)}$, where $H_{i}=G_{i}$ when the 
trader is naive and $H_{i}=\left(G_{i}, p\right)$ when they are rational. Naive traders' $\alpha_{i}$ do not react to a change in the informativeness of the price, whereas rational traders' $\alpha_{i}$ increase when $p$ becomes more informative. Consequently, rational traders transact more aggressively, imparting more variance to the price. In other words, the valuations that the price averages (the $v_{i}$ ) become more precise and consequently more volatile since they absorb part of the information of the other signals. The net effect on the equilibrium price is not defined a priori and can well be some endogenous volatility. However, this does not imply that a transaction tax would reduce price volatility or increase the welfare of liquidity traders.

\subsection{Equilibrium with naive traders}

When the traders are naive, $v_{i}=E\left[x \mid G_{i}\right]=\rho \sigma_{x} G_{i}$ and $\operatorname{var}\left(x \mid G_{i}\right)=\sigma_{x}^{2}\left(1-\rho^{2}\right)$, all $i$, and $\theta^{-1}-n=$ $\kappa \sigma_{x}^{2}\left(1-\rho^{2}\right)$. Using $p=\theta \sum_{i=1}^{n} v_{i}+(1-n \theta) \varepsilon$, and $p=a \sum_{i=1}^{n} G_{i}+b \varepsilon$, we get

$$
\begin{aligned}
a^{N} & =\frac{\rho \sigma_{x}}{n+\kappa \sigma_{x}^{2}\left(1-\rho^{2}\right)} \\
b^{N} & =\frac{\kappa \sigma_{x}^{2}\left(1-\rho^{2}\right)}{n+\kappa \sigma_{x}^{2}\left(1-\rho^{2}\right)} \\
\beta^{N} & =\kappa \sigma_{x} \frac{\left(1-\rho^{2}\right)}{\rho}
\end{aligned}
$$

When traders are naive, the equilibrium $\beta^{N}$ is independent of the number of traders, which implies that the price informativeness is increasing in $n$. It also implies that the properties of $\beta$ and $I_{p}^{N}$ derived with two traders still hold. The upper bound of the set of admissible values for $n$ - those for which the covariance matrix between the $G_{i}$ 's and $x$ is positive definite - is $\frac{1}{\rho^{2}}$. The price variance is $v a r_{p}=\frac{\left(\rho \sigma_{x}\right)^{2} n+\left[\kappa \sigma_{x}^{2}\left(1-\rho^{2}\right)\right]^{2} \sigma_{\varepsilon}^{2}}{\left[n+\kappa \sigma_{x}^{2}\left(1-\rho^{2}\right)\right]^{2}}$. It remains finite even when $n$ attains its upper bound. The sign of $\frac{\partial}{\partial n} \operatorname{var}_{p}$ is not determined a priori, but, is negative for $n \geq \kappa \sigma_{x}^{2}\left(1-\rho^{2}\right)$.

\subsection{Equilibrium with rational traders}

Using the same reasoning as with two traders, we get:

$$
\begin{aligned}
v_{1} & =E\left[x \mid G_{1}, p\right] \\
& =E\left[x \mid G_{1}, a\left(G_{1}+\ldots+G_{n}\right)+b \varepsilon\right] \\
& =E\left[x \mid G_{1}, G_{2}+\ldots+G_{n}+\beta \varepsilon\right] \\
& =E\left[x \mid G_{1}\right]+E\left[x \mid G_{2}+\ldots+G_{n}+\beta \varepsilon\right] \\
& =\sigma_{x} \rho G_{1}+\frac{\operatorname{cov}\left(x, G_{2}+\ldots+G_{n}+\beta \varepsilon\right)}{\operatorname{var}\left[G_{2}+\ldots+G_{n}+\beta \varepsilon\right]}\left(G_{2}+\ldots+G_{n}+\beta \varepsilon\right) \\
& =\sigma_{x} \rho G_{1}+\frac{(n-1) \sigma_{x} \rho}{(n-1)+\beta^{2} \sigma_{\varepsilon}^{2}}\left(G_{2}+\ldots+G_{n}+\beta \varepsilon\right)
\end{aligned}
$$


Consequently,

$$
\sum_{i=1}^{n} v_{i}=\sigma_{x} \rho\left(1+\frac{(n-1)^{2}}{(n-1)+\beta^{2} \sigma_{\varepsilon}^{2}}\right)\left(\sum_{i=1}^{n} G_{i}\right)+\frac{n(n-1) \sigma_{x} \rho}{(n-1)+\beta^{2} \sigma_{\varepsilon}^{2}} \beta \varepsilon
$$

To use $\theta^{-1} p=\sum_{i=1}^{n} v_{i}+\left(\theta^{-1}-n\right) \varepsilon$, note that $\theta^{-1}-n=\kappa\left(\sigma_{x}^{2}-E\left[v_{1}^{2}\right]\right)$ and

$$
\begin{aligned}
E\left[v_{1}^{2}\right] & =E\left[E\left[x \mid G_{1}\right]^{2}\right]+E\left[E\left[x \mid G_{2}+\ldots+G_{n}+\beta \varepsilon\right]^{2}\right] \\
& =\left(\sigma_{x} \rho\right)^{2}+\frac{\left[\operatorname{cov}\left(x, \sum_{i=2}^{n} G_{j}+\beta \varepsilon\right)\right]^{2}}{\operatorname{var}\left(\sum_{i=1}^{n} G_{j}+\beta \varepsilon\right)} \\
& =\left(\sigma_{x} \rho\right)^{2}+\frac{(n-1)^{2}\left(\sigma_{x} \rho\right)^{2}}{(n-1)+\beta^{2} \sigma_{\varepsilon}^{2}} \\
& =\left(\sigma_{x} \rho\right)^{2} \frac{n(n-1)+\beta^{2} \sigma_{\varepsilon}^{2}}{(n-1)+\beta^{2} \sigma_{\varepsilon}^{2}}
\end{aligned}
$$

Hence $\theta^{-1}-n=\kappa \sigma_{x}^{2} \frac{(n-1)\left(1-\rho^{2} n\right)+\left(1-\rho^{2}\right) \beta^{2} \sigma_{\varepsilon}^{2}}{n-1+\beta^{2} \sigma_{\varepsilon}^{2}}$. Using $p=a \sum_{i=1}^{n} G_{i}+b \varepsilon$ and $p=\sum_{i=1}^{n} \theta_{i} v_{i}+\theta_{n+1} \varepsilon$, we get

$$
\beta=\frac{\rho n(n-1) \beta+\kappa \sigma_{x}\left[(n-1)\left(1-n \rho^{2}\right)+\left(1-\rho^{2}\right) \beta^{2} \sigma_{\varepsilon}^{2}\right]}{\rho\left[n(n-1)+\beta^{2} \sigma_{\varepsilon}^{2}\right]}
$$

Or, equivalently,

$$
u\left(\beta, \rho, \kappa \sigma_{x}\right)=v\left(n, \rho, \kappa \sigma_{x}, \sigma_{\varepsilon}\right)
$$

with

$$
\begin{aligned}
& u\left(\beta, \rho, \kappa \sigma_{x}\right)=\rho \beta^{3}-\kappa \sigma_{x}\left(1-\rho^{2}\right) \beta^{2} \\
& v\left(n, \rho, \kappa \sigma_{x}, \sigma_{\varepsilon}\right)=\frac{\kappa \sigma_{x}}{\sigma_{\varepsilon}^{2}}(n-1)\left(1-n \rho^{2}\right)
\end{aligned}
$$

Since $n<\frac{1}{\rho^{2}}, n \geq 2$, and $\kappa \sigma_{x}>0$, the function $v$ is always positive. The function $u$ is the same as in the section on the equilibrium with two informed traders. Consequently, the $\beta$ that solves (18) is superior to $\kappa \sigma_{x} \frac{1-\rho^{2}}{\rho}$, which is the value of $\beta$ when traders are naive. The function $v$, and hence $\beta$, are increasing in $n$ for $n<\frac{1+\rho^{2}}{2 \rho^{2}}$ and decreasing for $n \geq \frac{1+\rho^{2}}{2 \rho^{2}}$. This implies that $I_{p}$ is increasing in $n$ when $n \geq \frac{1+\rho^{2}}{2 \rho^{2}}$, since $\frac{\partial I_{p}}{\partial n}>0$ and $\frac{\partial I_{p}}{\partial \beta}<0$. Numerical examples showed that the price informativeness was also increasing in $n$ for $n<\frac{1+\rho^{2}}{2 \rho^{2}}$. As $n \rightarrow \frac{1}{\rho^{2}}, v \rightarrow 0$ and $\beta \rightarrow \kappa \sigma_{x} \frac{1-\rho^{2}}{\rho}$. As a consequence the price informativeness is always lower with rational than with naive traders and the two converge to the same value as the number of traders tends to its upper bound. The same reasoning as for the case with two traders shows that the $\beta$ is decreasing in $\rho$ and $\sigma_{\varepsilon}$, and increasing in $\kappa$ and $\sigma_{x}$. As in the two-trader case, the price informativeness is increasing in $\rho$, and decreasing in $\kappa, \sigma_{x}$ and $\sigma_{\varepsilon}$ (the proofs are similar), while the price variance depends on $a$ and $b$, which can be computed as functions of the equilibrium $\beta$. As before, the $R$ superscript denotes the 
case when traders are rational.

$$
\begin{aligned}
a^{R} & =\frac{\sigma_{x} \rho\left[n(n-1)+\beta^{2} \sigma_{\varepsilon}^{2}\right]}{n\left(n-1+\beta^{2} \sigma_{\varepsilon}^{2}\right)+\kappa \sigma_{x}^{2}\left((n-1)\left(1-n \rho^{2}\right)+\left(1-\rho^{2}\right) \beta^{2} \sigma_{\varepsilon}^{2}\right)} \\
b^{R} & =\frac{\rho \sigma_{x} n(n-1) \beta+\kappa \sigma_{x}^{2}\left((n-1)\left(1-n \rho^{2}\right)+\left(1-\rho^{2}\right) \beta^{2} \sigma_{\varepsilon}^{2}\right)}{n\left(n-1+\beta^{2} \sigma_{\varepsilon}^{2}\right)+\kappa \sigma_{x}^{2}\left((n-1)\left(1-n \rho^{2}\right)+\left(1-\rho^{2}\right) \beta^{2} \sigma_{\varepsilon}^{2}\right)}
\end{aligned}
$$

Equations (20) and (14) show that $a^{R}>a^{N}$ for $n \geq 2$. This implies that $b^{R}>b^{N}$ since $\beta^{R}>\beta^{N}$. As a consequence, the price variance is higher when traders are rational than when they are naive. Although $\beta^{R}$ converges to $\beta^{N}$ when $n \rightarrow \frac{1}{\rho^{2}}, a^{R}$ and $b^{R}$ don't converge to $a^{N}$ and $b^{N}$.

\subsection{Equilibrium with transaction costs}

In this section, some impediments to trade are introduced in the market, such as a proportional tax on profit and a transaction cost (which can also be thought of as a transaction tax). These costs are imposed on the informed traders but not on the liquidity trader, partly because proponents of a transaction tax, for example, pretend to defend liquidity traders, not to increase their costs. Another reason is that the liquidity trader's demand, although price-sensitive, does not stem from maximizing a proper utility function, so that we cannot predict how it will react to changes in transaction costs. In the following, we will examine two types of frictions: a proportional tax on profit, a cost quadratic in the amount transacted.

A proportional tax on profits at a rate $\tau$ can be accommodated in the model by replacing $\lambda$, the risk aversion coefficient of the informed trader's, by $\lambda(1-\tau)$. Consequently, when traders are naive and when they are rational, introducing such a tax or increasing the tax rate decreases not only the volatility of price but also its informativeness. ${ }^{8}$

In the remainder, we assume that there are $n$ traders, with $n \geq 2$, and that their signals are identically and independently distributed. A transaction cost is introduced that is quadratic in the order size, ${ }^{9}$ the total cost is $\frac{1}{2} c y_{i}^{2}$ where $y_{i}$ is trader $i$ 's demand and $c$ is called the cost coefficient. With $H_{i}=G_{i}$ when informed traders are naive and $H_{i}=\left(G_{i}, p\right)$ when they are rational, trader $i$ 's objective function is now

$$
E\left[y_{i}(x-p) \mid H_{i}\right]-\frac{1}{2} \lambda y_{i}^{2} \operatorname{var}\left(x \mid H_{i}\right)-\frac{c}{2} y_{i}^{2}
$$

\footnotetext{
${ }^{8}$ The results have been obtained analytically for the price informativeness and numerically for the price variance.

${ }^{9}$ Letting the transaction cost be proportional to the order size $\left|y_{i}\right|$ would result in a non-differentiable objective function and make the demand function intractable.
} 
The optimal demand function is

$$
y_{i}=\frac{E\left[x \mid H_{i}\right]-p}{\lambda \operatorname{var}\left(x \mid H_{i}\right)+c}
$$

Replacing $y_{i}$ by its value in (22) shows that the optimized objective function in equation (21) is never negative, therefore, the informed trader will always remain in the market (assuming the alternative to trade is to do, and gain, nothing). As before, assuming that the informed traders' private signals are independently and identically distributed, the market-clearing price $p$ is such that $p=\theta \sum_{i=1}^{n} v_{i}+(1-n \theta) \varepsilon$, where $\left.\theta^{-1}=s\left(\lambda \operatorname{var}\left(x \mid H_{i}\right)\right)+c\right)+n$.

\subsubsection{Equilibrium with naive traders}

When traders are naive $v_{i}=E\left[x \mid G_{i}\right]=\sigma_{x} \rho G_{i}$ and $\operatorname{var}\left[x \mid G_{i}\right]=\sigma_{x}^{2}\left(1-\rho^{2}\right)$, which implies that

$$
\begin{aligned}
a^{N} & =\frac{\sigma_{x} \rho}{n+\lambda s \sigma_{x}^{2}\left(1-\rho^{2}\right)+c s} \\
b^{N} & =\frac{\lambda s \sigma_{x}^{2}\left(1-\rho^{2}\right)+c s}{n+\lambda s \sigma_{x}^{2}\left(1-\rho^{2}\right)+c s} \\
\beta^{N} & =\frac{\lambda s \sigma_{x}^{2}\left(1-\rho^{2}\right)+c s}{\sigma_{x} \rho}
\end{aligned}
$$

This shows that $\beta^{N}$ is an increasing function of the cost coefficient, $c$, and therefore that the price informativeness $I_{p}^{N}$ is a decreasing function of $c$. From what precedes, it can be proven that the sign of $\frac{\partial}{\partial c} \operatorname{var}_{p}$ is increasing in $c, \lambda, s, \sigma_{\varepsilon}$, and decreasing in $\rho$. Hence, the likelihood that an increase in the transaction cost factor $c$ would boost the price variance is increasing in the informed traders' risk aversion, the price sensitivity of the liquidity demand, the volatility of the liquidity shock, and decreasing in the precision of the information of the informed traders. Also raising the cost above some threshold (depending on the other parameters) will increase the volatility of the price. Figures (5) and (4) show the variance and informativeness of the price when the transaction cost coefficient $c$ increases and $\rho=.25$ and $\kappa=\sigma_{x}=\sigma_{\varepsilon}=1$.

\subsubsection{Equilibrium with rational traders}

Using $p=a \sum_{i=1}^{n} G_{i}+b \varepsilon=\theta \sum_{i=1}^{n} v_{i}+(1-n \theta) \varepsilon$, and $\theta^{-1}-n=\lambda s \sigma_{x}^{2} \frac{(n-1)\left(1-n \rho^{2}\right)+\left(1-\rho^{2}\right) \beta^{2} \sigma_{\varepsilon}^{2}}{n-1+\beta^{2} \sigma_{\varepsilon}^{2}}+c s$, we get

$$
\begin{aligned}
\theta^{-1} p & =\sigma_{x} \rho \frac{n(n-1)+\beta^{2} \sigma_{\varepsilon}^{2}}{n-1+\beta^{2} \sigma_{\varepsilon}^{2}} \sum_{i=1}^{n} G_{i} \\
& +\frac{\rho n(n-1) \sigma_{x} \beta+\lambda s \sigma_{x}^{2}\left[(n-1)\left(1-n \rho^{2}\right)+\left(1-\rho^{2}\right) \beta^{2} \sigma_{\varepsilon}^{2}\right]+\left(n-1+\beta^{2} \sigma_{\varepsilon}^{2}\right) c s}{n-1+\beta^{2} \sigma_{\varepsilon}^{2}} \varepsilon
\end{aligned}
$$


It follows from above that the equilibrium $\beta$ solves the following equation

$$
\beta=\frac{\rho \sigma_{x} n(n-1) \beta+\lambda s \sigma_{x}^{2}(n-1)\left(1-n \rho^{2}\right)+\left(\lambda s \sigma_{x}^{2}\left(1-\rho^{2}\right)+c s\right) \beta^{2} \sigma_{\varepsilon}^{2}+(n-1) c s}{\sigma_{x} \rho\left(n(n-1)+\beta^{2} \sigma_{\varepsilon}^{2}\right)}
$$

Equivalently, the equilibrium $\beta$ solves

$$
u\left(\beta, c, \rho, \lambda, s, \sigma_{x}\right)=v\left(c, \rho, n, \lambda, s, \sigma_{\varepsilon}, \sigma_{x}\right)
$$

with

$$
\begin{aligned}
& u\left(\beta, c, \rho, \lambda, s, \sigma_{x}\right)=\rho \sigma_{x} \beta^{3}-s\left(\lambda \sigma_{x}^{2}\left(1-\rho^{2}\right)+c\right) \beta^{2} \\
& v\left(c, \rho, n, \lambda, s, \sigma_{\varepsilon}, \sigma_{x}\right)=\frac{(n-1) s}{\sigma_{\varepsilon}^{2}}\left(\lambda \sigma_{x}^{2}\left(1-n \rho^{2}\right)+c\right)
\end{aligned}
$$

The equilibrium $\beta$ lies at the intersection of $u$ and $v$. Increasing $c \operatorname{shifts} u$ to the right and $v$ down, driving $\beta$ up. It follows that the price informativeness is decreasing in $c$ since it is a decreasing function of $\beta$ and depends in $c$ only through $\beta$. The price variance depends also on $c$ only through $a$ and $b$, which as functions of $\beta$ are as follows.

$$
\begin{aligned}
a^{R} & =\frac{\sigma_{x} \rho\left[n(n-1)+\beta^{2} \sigma_{\varepsilon}^{2}\right]}{(n+s c)\left(n-1+\beta^{2} \sigma_{\varepsilon}^{2}\right)+\lambda s \sigma_{x}^{2}\left[(n-1)\left(1-\rho^{2} n\right)+\left(1-\rho^{2}\right) \beta^{2} \sigma_{\varepsilon}^{2}\right]} \\
b^{R} & =\frac{n(n-1) \sigma_{x} \rho \beta+\lambda s \sigma_{x}^{2}\left[(n-1)\left(1-n \rho^{2}\right)+\left(1-\rho^{2}\right) \beta^{2} \sigma_{\varepsilon}^{2}\right]+\left(n-1+\sigma_{\varepsilon}^{2} \beta^{2}\right) c s}{(n+s c)\left(n-1+\beta^{2} \sigma_{\varepsilon}^{2}\right)+\lambda s \sigma_{x}^{2}\left[(n-1)\left(1-\rho^{2} n\right)+\left(1-\rho^{2}\right) \beta^{2} \sigma_{\varepsilon}^{2}\right]}
\end{aligned}
$$

The sign of $\frac{\partial}{\partial c} \operatorname{var}_{p}$ depends on the values of the other parameters, in particular $n$, which did not appear in the naive case. Numerical examples confirm the results obtained assuming traders are naive. Introducing a transaction cost tends to lead to an increase in the price variance, the more risk averse the informed traders are, the less precise their information is, the more price sensitive the liquidity traders are, and the more volatile their shock is. Figures (7) and (6) show the variance and informativeness of the price when the transaction cost coefficient $c$ increases and $\rho=.25$ and $\kappa=\sigma_{x}=\sigma_{\varepsilon}=1$. The effect of increasing $c$ on the price informativeness seems to be fairly similar when traders are naive and when they are rational, whereas the effect on the price variance is very different. When traders are rational, a transaction tax might bring volatility down. Be the informed traders naive or rational, increasing the transaction tax could lower liquidity trader's expected consumer surplus, $\frac{s}{2} E\left[(\varepsilon-p)^{2}\right]$, (see Figures (8) and $(9)$ ).

Hence, contrary to the view that "throwing some sand" in the market's wheels will control "excessive volatility", a transaction tax could well heighten market's volatility. The only certain consequence from the model of such a policy would be to make markets less informative. Kupiec 
(1996) had already shown that introducing a transaction tax could have perverse effects. Issues related to a securities transaction tax are reviewed in Hammond (1995). The contribution of the paper to this debate is to point out to similar results imposing as little structure as possible; Kupiec's results are based on a more complex, overlapping-generation model.

\subsection{Expected Trading Volume}

The trading volume is $z=\sum_{i=1}^{n+1} y_{i}^{+}$where the plus superscript indicate the positive truncation and $y_{n+1}$ is the liquidity trader's demand. Since the market clears, $y_{n+1}=-\sum_{i=1}^{n} y_{i}$. Recall that if $u \sim N\left(0, \sigma_{u}^{2}\right), E\left[u^{+}\right]=\frac{\sigma_{u}}{\sqrt{2 \pi}}$. Hence,

$$
E[z]=(2 \pi)^{-\frac{1}{2}}\left[\sum_{i=1}^{n} \sqrt{\operatorname{var}\left(y_{i}\right)}+\sqrt{\operatorname{var}\left(\sum_{i=1}^{n} y_{i}\right)}\right]
$$

Since the informed signals are identically distributed, the variance of $v_{i}-p$ and the covariance between $v_{i}-p$ and $v_{j}-p$ do not depend on $i$ and $j$. Let $\Phi=E\left[\left(v_{i}-p\right)^{2}\right]$ and $\Psi=E\left[\left(v_{i}-p\right)\left(v_{j}-p\right)\right]$, for $i \neq j$. Since $\operatorname{var}\left(\sum_{i=1}^{n} y_{i}\right)=\sum_{i=1}^{n} \operatorname{var}\left(y_{i}\right)+2 \sum_{i=1}^{n} \sum_{j>i} \operatorname{cov}\left(y_{i}, y_{j}\right)=\frac{n \Phi+n(n-1) \Psi}{\left(\operatorname{\lambda var}\left(x \mid H_{i}\right)\right)^{2}}$

$$
E[z]=\frac{n \sqrt{\Phi}+\sqrt{n \Phi+n(n-1) \Psi}}{\lambda \operatorname{var}\left(x \mid H_{i}\right) \sqrt{2 \pi}}
$$

Figure (10) displays the expected traded volume when traders are naive and when they are rational and how it reacts to an increase in the number of traders, for some values of parameters. Assuming that traders are rational instead of naive affects the expected volume in two ways. The fact that $\operatorname{var}\left(x \mid G_{i}, p\right) \leq \operatorname{var}\left(x \mid G_{i}\right)$ tends to increase $E[z]$. However, numerical examples-varying values for $n$ and $\rho$-point to $E\left[\left(E\left[x \mid G_{i}\right]-p\right)^{2}\right]>E\left[\left(E\left[x \mid G_{i}, p\right]-p\right)^{2}\right]$, and $E\left[\left(E\left[x \mid G_{i}\right]-p\right)\left(E\left[x \mid G_{j}\right]-p\right)\right]>$ $E\left[\left(E\left[x \mid G_{i}, p\right]-p\right)\left(E\left[x \mid G_{j}, p\right]-p\right)\right]$. Intuitively, since $E\left[x \mid G_{i}, p\right]$ incorporates information contained in $p$, it should be statistically "closer" to $p$ than $E\left[x \mid G_{i}\right]$ is. This should lead to a smaller expected volume when traders are rational. On net, in the numerical examples, this effect dominated and the expected volume was always smaller when assuming traders were rational. In other words, $E[z]$ is increasing in the variance of the demands of the informed traders and decreasing in the covariance between their demands and the equilibrium price. Because rational traders incorporate the market price into their information, the variance of the informed traders' demands is higher when traders are rational than when they are naive, but so is the the covariance between their demands and the market-clearing price. On net, the increase in their covariance with price seems to more than 
offset the increase in their variance, resulting in a lower expected trading volume when traders are rational.

\section{Equilibrium with multiple informed traders with correlated sig- nals}

When modeling a market with multiple informed traders, many researchers don't assume that the informed traders' private signals are independently and identically. Instead, they have each informed trader observe the true asset value, $x$, plus a measurement error composed of a systematic element $u$ and an idiosyncratic element $\eta_{i}$, independently distributed across traders. One advantage of this framework is that the number of informed traders can increase without bounds. More precisely, the private signal is

$$
G_{i}=x+u+\eta_{i}
$$

where the random variables $x, u$ and the $\eta_{i}$ are independently and jointly normally distributed with mean zero and respective variances $\sigma_{x}^{2}, \sigma_{u}^{2}, \sigma_{\eta}^{2}$. Hence, $E\left[x G_{i}\right]=\sigma_{x}^{2}, E\left[G_{i} G_{j}\right]=\sigma_{x}^{2}+\sigma_{u}^{2}$ when $i \neq j$, and $\mathrm{E}\left[G_{i}^{2}\right]=\sigma_{x}^{2}+\sigma_{u}^{2}+\sigma_{\eta}^{2}$. Since the informed traders' signals are still identically distributed, the equilibrium price is $p=a \sum_{i=1}^{n} G_{i}+b \varepsilon$.

As in the preceding sections, the price is more volatile and less informative when traders are rational than when they are naive. It can be proven that the price informativeness is increasing in $n$ when informed traders are naive and when they are rational and $\sigma_{u}=0$. When $\sigma_{u} \neq 0$, numerical examples point to the price informativeness to be increasing in $n$ as well (see Figures (11) and (12)). However, increasing the number of informed traders can lead to a more volatile price, even when traders are naive. This is due to the fact that the informed traders signals are correlated. The net effect of an increase in $n$ depends on the parameters $\sigma_{x}, \sigma_{u}, \sigma_{\eta}$, and $n$ itself. In the numerical examples used, when the variance of the price increases in $n$ for naive traders, it increases even more for rational traders, to converge to a higher limit (see Figures (13) and (14)).

Unless indicated otherwise, we keep the same notations as in the preceding section. When the informed traders are naive and when they are rational, the variance of the price and its informativeness are respectively

$$
\begin{aligned}
\operatorname{var}_{p} & =a^{2} n\left(\sigma_{\eta}^{2}+n\left(\sigma_{x}^{2}+\sigma_{u}^{2}\right)\right)+b^{2} \sigma_{\varepsilon}^{2} \\
I_{p} & =\frac{n^{2} \sigma_{x}^{2}}{n\left(\sigma_{\eta}^{2}+n\left(\sigma_{x}^{2}+\sigma_{u}^{2}\right)\right)+\beta^{2} \sigma_{\varepsilon}^{2}}
\end{aligned}
$$


Proposition 6 The equilibrium price is more volatile and less informative when traders are rational than when they are naive. This is because $a^{R}>a^{N}, b^{R}>b^{N}$, and $\beta^{R}>\beta^{N}$.

Proposition 7 Both when informed traders are rational and when they are naive, the informativeness of the equilibrium price is increasing in $\sigma_{x}$ and decreasing in $\sigma_{u}, \sigma_{\eta}$ and $\kappa$.

Proofs of proposition (6) and (7) are presented further below.

\subsection{Naive traders}

When traders are naive, their valuations are $v_{i}=E\left[x \mid G_{i}\right]=\frac{\sigma_{x}^{2}}{\sigma_{x}^{2}+\sigma_{u}^{2}+\sigma_{\eta}^{2}} G_{i}$. As a consequence,

$$
\begin{aligned}
a^{N} & =\frac{\sigma_{x}^{2}}{\left(\sigma_{x}^{2}+\sigma_{u}^{2}+\sigma_{\eta}^{2}\right) n+\kappa \sigma_{x}^{2}\left(\sigma_{u}^{2}+\sigma_{\eta}^{2}\right)}, \\
b^{N} & =\frac{\kappa \sigma_{x}^{2}\left(\sigma_{u}^{2}+\sigma_{\eta}^{2}\right)}{\left(\sigma_{x}^{2}+\sigma_{u}^{2}+\sigma_{\eta}^{2}\right) n+\kappa \sigma_{x}^{2}\left(\sigma_{u}^{2}+\sigma_{\eta}^{2}\right)}, \\
\beta^{N} & =\kappa\left(\sigma_{u}^{2}+\sigma_{\eta}^{2}\right) .
\end{aligned}
$$

The variance of the price and its informativeness are respectively

$$
\begin{aligned}
\operatorname{var}_{p}^{N} & =\frac{\sigma_{x}^{4}\left[\left(n \sigma_{\eta}^{2}+n^{2}\left(\sigma_{x}^{2}+\sigma_{u}^{2}\right)\right)+\kappa^{2}\left(\sigma_{u}^{2}+\sigma_{\eta}^{2}\right)^{2} \sigma_{\varepsilon}^{2}\right]}{\left[\left(\sigma_{x}^{2}+\sigma_{u}^{2}+\sigma_{\eta}^{2}\right) n+\kappa \sigma_{x}^{2}\left(\sigma_{u}^{2}+\sigma_{\eta}^{2}\right)\right]^{2}} \\
I_{p}^{N} & =\frac{n^{2} \sigma_{x}^{2}}{n \sigma_{\eta}^{2}+n^{2}\left(\sigma_{x}^{2}+\sigma_{u}^{2}\right)+\kappa^{2}\left(\sigma_{u}^{2}+\sigma_{\eta}^{2}\right)^{2} \sigma_{\varepsilon}^{2}} .
\end{aligned}
$$

The price precision is decreasing increasing in $n$ and $\sigma_{x}^{2}$, and decreasing in $\sigma_{\varepsilon}^{2}, \sigma_{u}^{2}, \sigma_{\eta}^{2}$ and $\kappa$. It is intuitive that increasing the variance of the noises, either stemming from the liquidity trader $(\varepsilon)$ or from the informed traders $(u$ and $\eta)$ will make the market price less informative. In contrast, increasing the number of traders introduces more information into the market. Besides, as in the previous sections, making the informed traders more risk averse or the liquidity trader more price sensitive limits the aggressiveness of the informed traders, who transfer less information to the market price. The effect of $\sigma_{x}$ differs from the preceding sections because the correlation between $x$ and $G_{i}, \frac{\sigma_{x}}{\sqrt{\sigma_{x}^{2}+\sigma_{u}^{2}+\sigma_{\eta}^{2}}}$, is an increasing function of $\sigma_{x}$. In the preceding sections, the correlation between $x$ and $G_{i}$ was independent of $\sigma_{x}$. Increasing the variance of the true value of the asset made the risk-averse informed trader less aggressive. Here, this effect is dominated by the increase in the correlation between $x$ and $G_{i}$.

Contrarily to the preceding sections, increasing the number of traders does not always reduce the price variance when the informed traders are naive. This comes from the fact that the traders' signals are correlated. It is easy to see that the sign of $\frac{\partial}{\partial n} \operatorname{var}_{p}^{N}$ depends, among others, on $n$. The 
correlation across signals also causes the price variance not to go to zero when $n$ tends to infinity. Comparing with the preceding sections, note that, although in both cases, $a^{N}$ and $b^{N}$ are of the order $\frac{1}{n}$ when $n$ tends to infinity, the coefficient of $\left(a^{N}\right)^{2}$ in the price variance is of order $n^{2}$ in the measurement-error case and of order $n$ when the traders' signals are independently distributed. Consequently, in the measurement-error framework, $\lim _{n \rightarrow \infty} \operatorname{var}_{p}^{N}=\left[\frac{\sigma_{x}^{2}}{\sigma_{x}^{2}+\sigma_{u}^{2}+\sigma_{\eta}^{2}}\right]^{2}\left(\sigma_{x}^{2}+\sigma_{u}^{2}\right)$ and $\lim _{n \rightarrow \infty} I_{p}^{N}=\frac{\sigma_{x}^{2}}{\sigma_{x}^{2}+\sigma_{u}^{2}}$.

\subsection{Rational traders}

When traders are rational, their valuations are $v_{i}=E\left[x \mid G_{i}, p\right]$. Consider the first informed trader and let $\mu=\frac{\sigma_{x}^{2}+\sigma_{u}^{2}}{\sigma_{x}^{2}+\sigma_{u}^{2}+\sigma_{\eta}^{2}}$, then $E\left[\left(G_{i}-\mu G_{1}\right) G_{1}\right]=0$, all $i \geq 2$.

$$
\begin{aligned}
v_{1} & =E\left[x \mid G_{1}, p\right] \\
& =E\left[x \mid G_{1}, a \sum_{i=1}^{n} G_{i}+b \varepsilon\right] \\
& =E\left[x \mid G_{1}, \sum_{i=2}^{n}\left(G_{i}-\mu G_{1}\right)+\beta \varepsilon\right] \\
& =E\left[x \mid G_{1}\right]+E\left[x \mid \sum_{i=2}^{n}\left(G_{i}-\mu G_{1}\right)+\beta \varepsilon\right] \\
& \left.=\frac{E\left[x G_{1}\right]}{E\left[G_{i}^{2}\right]} G_{i}+\frac{\operatorname{cov}\left(x \sum_{i=2}^{n}\left(G_{i}-\mu G_{1}\right)\right.}{\operatorname{var}\left(\sum_{i=2}^{n}\left(G_{i}-\mu G_{1}\right)+\beta \varepsilon\right)}\left(\sum_{i=2}^{n}\left(G_{i}-\mu G_{1}\right)+\beta \varepsilon\right)\right) .
\end{aligned}
$$

This result comes from the fact that $x, G_{1}, \ldots, G_{n}, \varepsilon$ are jointly normally distributed and that $\left(G_{i}-\mu G_{1}\right)$ is orthogonal to $G_{i}$ for all $i \geq 2$, and $\varepsilon$ is orthogonal to $x$.

$$
\begin{aligned}
E\left[x \sum_{i=1}^{n}\left(G_{i}-\mu G_{1}\right)\right] & =(n-1) \mu \sigma_{x}^{2}, \\
& =(n-1) \frac{\sigma_{x}^{2} \sigma_{\eta}^{2}}{\sigma_{x}^{2}+\sigma_{u}^{2}+\sigma_{\eta}^{2}} .
\end{aligned}
$$

To compute the variance of $\left(\sum_{i=2}^{n}\left(G_{i}-\mu G_{1}\right)+\beta \varepsilon\right)$, note that, for $i, j \geq 2, E\left[\left(G_{i}-\mu G_{1}\right)\left(G_{j}-\right.\right.$ $\left.\left.\mu G_{1}\right)\right]=\mu \sigma_{\eta}^{2}$ if $i \neq j$ and $E\left[\left(G_{i}-\mu G_{1}\right)\left(G_{j}-\mu G_{1}\right)\right]=(1+\mu) \sigma_{\eta}^{2}$ if $i=j$. Using the fact that for $2 \leq i \leq n$ and $2 \leq j \leq n, " i=j^{\prime \prime}$ occurs $(n-1)$ times and " $i \neq j^{\prime \prime}$ occurs $(n-1)(n-2)$ times, one gets

$$
\begin{aligned}
\operatorname{var}\left(\sum_{i=2}^{n}\left(G_{i}-\mu G_{1}\right)\right) & =\sum_{i=2}^{n} \sum_{j=2}^{n} E\left[\left(G_{i}-\mu G_{1}\right)\left(G_{j}-\mu G_{1}\right)\right] \\
& =[(n-1)(1+\mu)+(n-1)(n-2) \mu] \sigma_{\eta}^{2}, \\
& =\frac{(n-1) \sigma_{\eta}^{2}\left(n\left(\sigma_{x}^{2}+\sigma_{u}^{2}\right)+\sigma_{\eta}^{2}\right)}{\sigma_{x}^{2}+\sigma_{u}^{2}+\sigma_{\eta}^{2}}
\end{aligned}
$$


Equations (32), (33), (34) imply that

$$
\begin{aligned}
v_{1} & =\frac{\sigma_{x}^{2}}{\sigma_{x}^{2}+\sigma_{u}^{2}+\sigma_{\eta}^{2}} G_{1}+\frac{(n-1) \sigma_{x}^{2} \sigma_{\eta}^{2}}{\sigma_{\eta}^{2}(n-1)\left(n\left(\sigma_{x}^{2}+\sigma_{u}^{2}\right)+\sigma_{\eta}^{2}\right)+\left(\sigma_{x}^{2}+\sigma_{u}^{2}+\sigma_{\eta}^{2}\right) \beta^{2} \sigma_{\varepsilon}^{2}}\left[\sum_{i=2}^{n}\left(G_{i}-\mu G_{1}\right)+\beta \varepsilon\right] \\
& =\frac{\sigma_{x}^{2}\left((n-1) \sigma_{\eta}^{2}+\beta^{2} \sigma_{\varepsilon}^{2}\right) G_{1}+(n-1) \sigma_{x}^{2} \sigma \eta^{2}\left(\sum_{i=2}^{n} G_{i}+\beta \varepsilon\right)}{\sigma_{\eta}^{2}(n-1)\left(n\left(\sigma_{x}^{2}+\sigma_{u}^{2}\right)+\sigma_{\eta}^{2}\right)+\left(\sigma_{x}^{2}+\sigma_{u}^{2}+\sigma_{\eta}^{2}\right) \beta^{2} \sigma_{\varepsilon}^{2}} .
\end{aligned}
$$

Hence,

$$
\sum_{i=1}^{n} v_{i}=\frac{\sigma_{x}^{2}\left(n(n-1) \sigma_{\eta}^{2}+\beta^{2} \sigma_{\varepsilon}^{2}\right) \sum_{i=1}^{n} G_{i}+n(n-1) \sigma_{x}^{2} \sigma_{\eta}^{2} \beta \varepsilon}{\sigma_{\eta}^{2}(n-1)\left(n\left(\sigma_{x}^{2}+\sigma_{u}^{2}\right)+\sigma_{\eta}^{2}\right)+\left(\sigma_{x}^{2}+\sigma_{u}^{2}+\sigma_{\eta}^{2}\right) \beta^{2} \sigma_{\varepsilon}^{2}} .
$$

Using the fact that $\theta^{-1} p=\sum_{i=1}^{n} v_{i}+\left(\theta^{-1}-n\right) \varepsilon$, with

$$
\theta^{-1}-n=\kappa \sigma_{x}^{2} \frac{(n-1)\left(\sigma_{\eta}^{2}+n \sigma_{u}^{2}\right) \sigma_{\eta}^{2}+\left(\sigma_{\eta}^{2}+\sigma_{u}^{2}\right) \beta \sigma_{\varepsilon}^{2}}{\sigma_{\eta}^{2}(n-1)\left(n\left(\sigma_{x}^{2}+\sigma_{u}^{2}\right)+\sigma_{\eta}^{2}\right)+\left(\sigma_{x}^{2}+\sigma_{u}^{2}+\sigma_{\eta}^{2}\right) \beta^{2} \sigma_{\varepsilon}^{2}}
$$

and that, by definition, the ratio of the coefficient of $\varepsilon$ to that of $\left(\sum_{i=1}^{n} G_{i}\right)$ is equal to $\beta$, one gets:

$$
\beta=\frac{n(n-1) \sigma_{\eta}^{2} \beta+\kappa\left[(n-1)\left(\sigma_{\eta}^{2}+n \sigma_{u}^{2}\right) \sigma_{\eta}^{2}+\left(\sigma_{\eta}^{2}+\sigma_{u}^{2}\right) \beta^{2} \sigma_{\varepsilon}^{2}\right]}{\sigma_{\eta}^{2} n(n-1)+\beta^{2} \sigma_{\varepsilon}^{2}} .
$$

The equilibrium $\beta$ must solve the following cubic equation:

$$
\varphi\left(\beta, \sigma_{\eta}, \sigma_{u}, \sigma_{\varepsilon}, \kappa, n\right)=\sigma_{\varepsilon}^{2} \beta^{3}-\kappa \sigma_{\varepsilon}^{2}\left(\sigma_{\eta}^{2}+\sigma_{u}^{2}\right) \beta^{2}-\kappa \sigma_{\eta}^{2}(n-1)\left(\sigma_{\eta}^{2}+n \sigma_{u}^{2}\right)
$$

Or equivalently,

$$
u\left(\beta, \sigma_{\eta}, \sigma_{u}, \kappa, n\right)=v\left(\sigma_{\eta}, \sigma_{u}, \sigma_{\varepsilon}, \kappa, n\right),
$$

with

$$
\begin{aligned}
& u\left(\beta, \sigma_{\eta}, \sigma_{u}, \kappa, n\right)=\beta^{2}\left(\beta-\kappa\left(\sigma_{\eta}^{2}+\sigma_{u}^{2}\right)\right) \\
& v\left(\sigma_{\eta}, \sigma_{u}, \sigma_{\varepsilon}, \kappa, n\right)=\kappa \frac{\sigma_{\eta}^{2}}{\sigma_{\varepsilon}^{2}}(n-1)\left(\sigma_{\eta}^{2}+n \sigma_{u}^{2}\right) .
\end{aligned}
$$

For all values of the parameters $\sigma_{\eta}, \sigma_{u}, \kappa$, and $n$, the function $u$ is negative or null for $\beta \leq \kappa\left(\sigma_{\eta}^{2}+\sigma_{u}^{2}\right)$ and positive for $\beta>\kappa\left(\sigma_{\eta}^{2}+\sigma_{u}^{2}\right)$. For $n \geq 2$, the function $v$ is always positive. Hence the $\beta$ that solves (39) is superior to $\kappa\left(\sigma_{\eta}^{2}+\sigma_{u}^{2}\right)$, which is the value of $\beta$ when traders are naive. Therefore, $\beta^{R}>\beta^{N}$, and the two coincide if $n=1, \sigma_{\eta}=0$ or $\kappa=0$, that is if there is only one informed trader, if there is no idiosyncratic measurement error, so that each informed trader observes the same signal, if the informed traders are risk neutral $(\lambda=0)$ or if there is no noise trader $(s=0)$.

Like in the sections above, using the implicit function theorem, one can show that the equilib- 
rium $\beta$ is increasing in $\sigma_{\eta}, \sigma_{u}$, and $\kappa$, which implies that the precision of the price is decreasing in those parameters. Using the implicit function theorem and the chain rule, one shows that $\sigma_{\varepsilon}^{2} \beta\left(\sigma_{\varepsilon}\right)$ is a increasing function function of $\sigma_{\varepsilon}$, therefore the price precision is decreasing in this parameter. Since $\beta^{R}$ is independent of $\sigma_{x}$, the price informativeness is increasing in $\sigma_{x}$, like in the naive-trader case. When $\sigma_{u}=0$, increasing the number of traders improves the price precision, but I could not establish the sign of $\frac{\partial I_{p}}{\partial n}$ when $\sigma_{u}>0$, although it was always positive in the numerical examples used. In equilibrium $a^{R}=A\left(\beta^{R}, \sigma_{\eta}, \sigma_{u}, \kappa, n\right)$, where

$A=\frac{\sigma_{x}^{2}\left(n(n-1) \sigma_{\eta}^{2}+\beta^{2} \sigma_{\varepsilon}^{2}\right)}{\left[\sigma_{\eta}^{2}(n-1)\left(n\left(\sigma_{x}^{2}+\sigma_{u}^{2}\right)+\sigma_{\eta}^{2}\right)+\left(\sigma_{x}^{2}+\sigma_{u}^{2}+\sigma_{\eta}^{2}\right) \beta^{2} \sigma_{\varepsilon}^{2}\right] n+\kappa \sigma_{x}^{2}\left[(n-1)\left(\sigma_{\eta}^{2}+n \sigma_{u}^{2}\right) \sigma_{\eta}^{2}+\left(\sigma_{\eta}^{2}+\sigma_{u}^{2}\right) \beta^{2} \sigma_{\varepsilon}^{2}\right]}$.

It can be shown that $A\left(\beta, \sigma_{\eta}, \sigma_{u}, \kappa, n\right)-a^{N}>0$ for all $\beta$. Hence, $a^{R}>a^{N}$ and $b^{R}>b^{N}$ since $\beta^{R}>\beta^{N}$.

The measurement-error framework makes it possible to consider any $n$ and hence to study the properties of the limit economy when $n$ goes to infinity. Examining the real root of the cubic equation (38) shows that, when $n \rightarrow \infty$,

$$
\frac{\beta}{[n(n-1)]^{\frac{1}{3}}} \sim\left[\frac{\kappa \sigma_{\eta}^{2}}{\sigma_{\varepsilon}^{2}}\left(\frac{1}{n} \sigma_{\eta}^{2}+\sigma_{u}^{2}\right)\right]^{\frac{1}{3}}
$$

Equation (42) implies that, when $n \rightarrow \infty$,

$$
\beta \sim\left[\frac{\kappa \sigma_{\eta}^{2} \sigma_{u}^{2}}{\sigma_{\varepsilon}^{2}}\right]^{\frac{1}{3}}[n(n-1)]^{\frac{1}{3}}
$$

when $\sigma_{u} \neq 0$, and

$$
\beta \sim\left[\frac{\kappa \sigma_{\eta}^{4}}{\sigma_{\varepsilon}^{2}}\right]^{\frac{1}{3}}(n-1)^{\frac{1}{3}}
$$

when $\sigma_{u}=0$. When $\sigma_{u} \neq 0$, as $n \rightarrow+\infty, a^{R} \sim \frac{\sigma_{x}^{2}}{\sigma_{x}^{2}+\sigma_{u}^{2}} \frac{1}{n}$ and $b^{R} \sim\left[\frac{\kappa \sigma_{\eta}^{2} \sigma_{u}^{2}}{\sigma_{\varepsilon}^{2}}\right]^{\frac{1}{3}} \frac{\sigma_{x}^{2}}{\sigma_{x}^{2}+\sigma_{u}^{2}}\left(\frac{1}{n}\right)^{\frac{1}{3}}$, which implies that

$$
p \sim \frac{\sigma_{x}^{2}}{\sigma_{x}^{2}+\sigma_{u}^{2}}(x+u)+\frac{\sigma_{x}^{2}}{\sigma_{x}^{2}+\sigma_{u}^{2}} \frac{1}{n} \sum_{i=1}^{n} \eta_{i}+\left[\frac{\kappa \sigma_{\eta}^{2} \sigma_{u}^{2}}{\sigma_{\varepsilon}^{2}}\right]^{\frac{1}{3}} \frac{\sigma_{x}^{2}}{\sigma_{x}^{2}+\sigma_{u}^{2}}\left(\frac{1}{n}\right)^{\frac{1}{3}} \varepsilon
$$


When $\sigma_{u}=0$, as $n \rightarrow+\infty, a^{R} \sim \frac{1}{n}$ and $b^{R} \sim\left[\frac{\kappa \sigma_{\eta}^{4}}{\sigma_{\varepsilon}^{2}}\right]^{\frac{1}{3}}\left(\frac{1}{n}\right)^{\frac{2}{3}}$, which implies that

$$
p \sim x+\frac{1}{n} \sum_{i=1}^{n} \eta_{i}+\left[\frac{\kappa \sigma_{\eta}^{4}}{\sigma_{\varepsilon}^{2}}\right]^{\frac{1}{3}}\left(\frac{1}{n}\right)^{\frac{2}{3}} \varepsilon
$$

When the informed traders are rational, $p \rightarrow \frac{\sigma_{x}^{2}}{\sigma_{x}^{2}+\sigma_{u}^{2}}(x+u)$ when $\sigma_{u} \neq 0$ and $p \rightarrow x$ when $\sigma_{u}=0$; when they are naive, $p \rightarrow \frac{\sigma_{x}^{2}}{\sigma_{x}^{2}+\sigma_{u}^{2}+\sigma_{\eta}^{2}}(x+u)$ when $\sigma_{u} \neq 0$ and $p \rightarrow \frac{\sigma_{x}^{2}}{\sigma_{x}^{2}+\sigma_{\eta}^{2}} x$ when $\sigma_{u}=0$. As the number of traders tends to infinity, the market price with naive informed traders reveals the same information as the one with rational informed traders. In both cases, the price converges to a multiple of $(x+u)$ when $\sigma_{u} \neq 0$ or a multiple of $x$ when $\sigma_{u}=0$. This multiple is always smaller when traders are naive, which makes the market price less volatile when traders are naive than when they are rational. Assuming rational instead of naive informed traders also affects the market price's rate of convergence. In particular, the coefficient on the liquidity noise $\varepsilon$ is of order $\frac{1}{n}$ when they are naive, of order $\left(\frac{1}{n}\right)^{\frac{1}{3}}$ when traders are rational and $\sigma_{u} \neq 0$, and of order $\left(\frac{1}{n}\right)^{\frac{2}{3}}$ when traders are rational and $\sigma_{u}=0$. In this way, the economy with rational informed traders filters the liquidity noise out of the equilibrium price less rapidly than the economy with naive traders, even more so when their signals contains a common measurement error. In other words, each rational traders, knowing that the other traders are rational too and will use the market price to infer some information about his signal, tends to hide behind the liquidity noise, which, in equilibrium, keeps more noise into the system. Rational traders' attempt to "learn from others" leads in equilibrium to a slower revelation of the aggregate information available in the economy $(x$ or $x+u)$ than would be the case with naive traders. Similar insights about the rate of learning taking place in competitive markets have been provided by Vives (1996). 


\section{Conclusion}

The paper attempts to capture the effect of the increase in the share of financial assets controlled by institutional investors - who should be more sophisticated than other market participantson the volatility and precision of the market price and how those variables evolve when market parameters change. The paper models institutional investors as rational and individual investors as naive, that is, the former extract information from the market price and the latter do not. Then, using a model with multiple informed traders and one liquidity trader, the paper first compares the equilibria when traders are naive and when they are rational, and studies how changes in the model parameters (the correlation of the informed traders' signals in the case of two informed traders, or, alternatively, the number of informed traders, and in all cases, the precision of their signal, their risk aversion, etc) affect the the variance of the price and its informativeness. The paper shows that the equilibrium price is always more volatile and less informative when traders are rational than when they are naive. Intuitively, rational traders hide behind the noise created by the liquidity trader, keeping more of this noise in market in equilibrium than naive traders would do. Results also point that, when traders are rational, an increase in the number of informed traders could lead to a surge in the price volatility, a problem introducing a transaction tax in the model does not seem to solve. 


\section{REFERENCES}

Brown, D., and Robert H. Jennings (1989), "On Technical Analysis," The Review of Financial Studies, Vol 2, No 4, p 527-551.

De Long, B., A. Shleifer, L. Summers, and R. Waldman, (1990), "Noise Trader Risk in Financial Markets," Journal of Political Economy, Vol 98, No. 4, pp. 703-738.

Diamond, D. and R. Verrechia (1981), "Information Aggregation in a Noisy Rational Expectation Economy," Journal of Financial Economics, vol 9, p 221-235.

Friedman, B. (1996), "Economic Implications of Changing Share Ownership," The Journal of Portfolio Management, Spring 1996, Vol 22. No 3. pp. 59-70.

Grossman, S. and J. Stiglitz (1980), "On the Impossibility of Informationally Efficient Markets," American Economic Review, 70, pp. 393-408.

Hammond, S. (1995) "Securities Transaction Tax, False Hopes and Unintended Consequences," Catalyst Institute, Irwin, edited by Suzanne Hammond.

Kennickel, A., M. Starr-McCluer, and A. Sundèn, (1997) "Family Finances in the U.S.: Recent Evidence from the Survey of Consumer Finances," Federal Reserve Bulletin, January 1997, pp. $1-24$.

Kupiec, P., "Noise Traders, Excess Volatility, and a Securities Transactions Tax," Journal of Financial Service Research, Vol 10, pp. 115-129.

Milgrom, P. and N. Stockey (1982), "Information, Trade and Common Knowledge," Journal of Economics Theory, No 26, p. 17-27.

Pagano, M. (1989), "Trading Volume and Asset Liquidity," Quarterly Journal of Economics, pp. 255-274.

Sias, R. (1996), "Volatility and the Institutional Investor," Financial Analysts Journal, March/April 1996. pp. 13-20.

Sias, R. (1997), "Price Pressure and the Role of Institutional Investors in Closed-Ends Funds," The Journal of Financial Research, Vol XX, No 2, pp. 211-229.

Vives, X. (1996), "Social Learning and Rational Expectations," European Economic Review 40, pp. 589-601. 


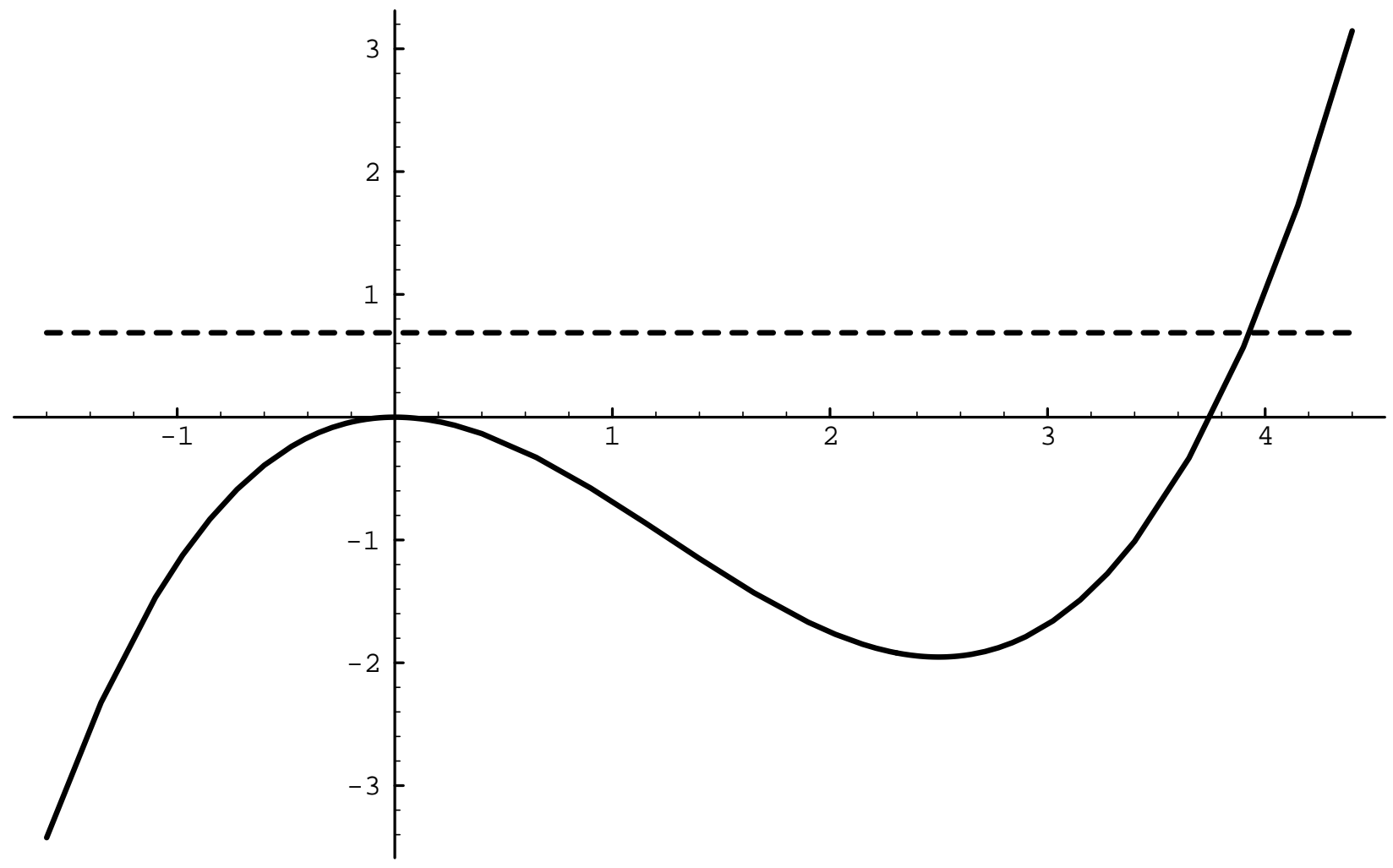

Figure 1: $v$ (solid line) and $u$ (dashed line) as a function of $\beta$, with $u\left(\beta, \rho, \kappa \sigma_{x}\right)=\rho \beta^{3}-\kappa \sigma_{x}(1-$ $\left.\rho^{2}\right) \beta^{2}$ and $v\left(\gamma, \sigma_{\varepsilon}^{2}, \rho, \kappa \sigma_{x}\right)=\frac{\kappa \sigma_{x}}{\sigma_{\varepsilon}^{2}}(1-\gamma)\left(\gamma+1-2 \rho^{2}\right) ; \rho=.5, \kappa=\sigma_{x}=\sigma_{\varepsilon}=1$. 


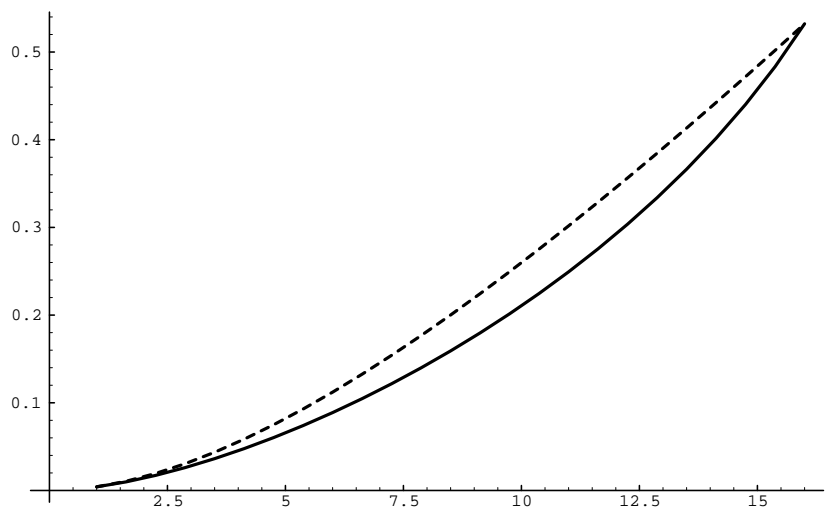

Figure 2: Price informativeness as a function of the number of informed traders when they are naive (dashed line) and when they are rational (solid line); $\rho=.25, \kappa=\sigma_{x}=\sigma_{\varepsilon}=1$.

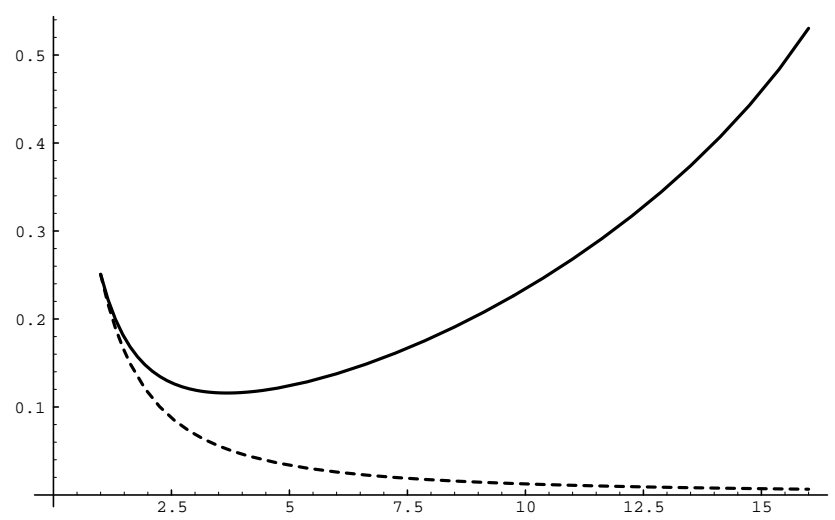

Figure 3: Price variance as a function of the number of informed traders when they are naive (dashed line) and when they are rational (solid line); $\rho=.25, \kappa=\sigma_{x}=\sigma_{\varepsilon}=1$. 


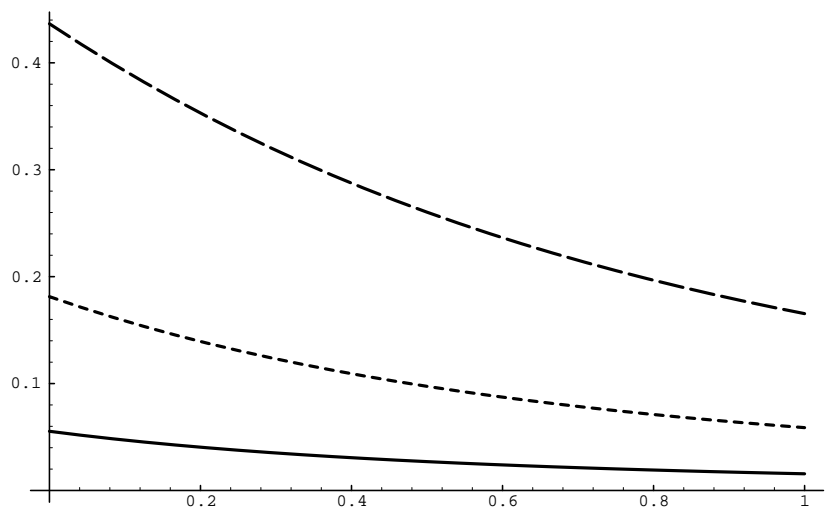

Figure 4: Price informativeness as a function of the transaction cost when informed traders are naive. The number of informed traders is set to 4 (solid line), 8 (short-dashed line) and 14 (long-dashed line); $\rho=.25, \kappa=\sigma_{x}=\sigma_{\varepsilon}=1$.

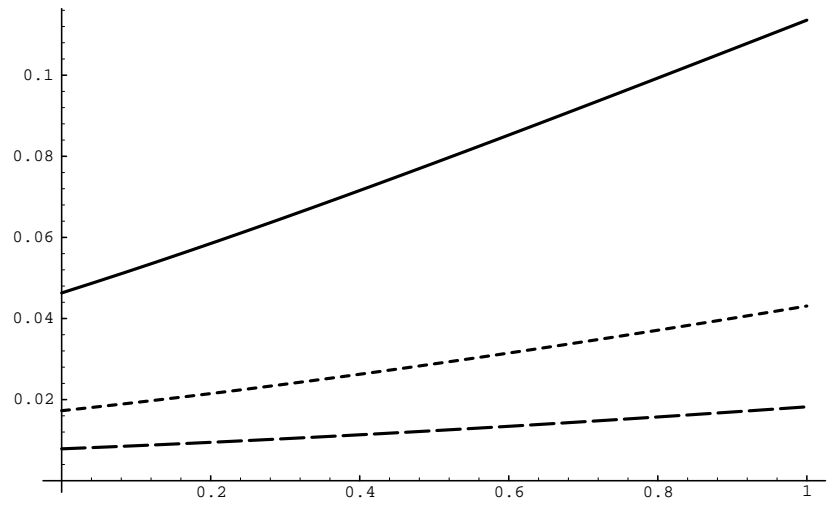

Figure 5: Price variance as a function of the transaction cost when informed traders are naive. The number of informed traders is set to 4 (solid line), 8 (short-dashed line) and 14 (long-dashed line); $\rho=.25, \kappa=\sigma_{x}=\sigma_{\varepsilon}=1$. 


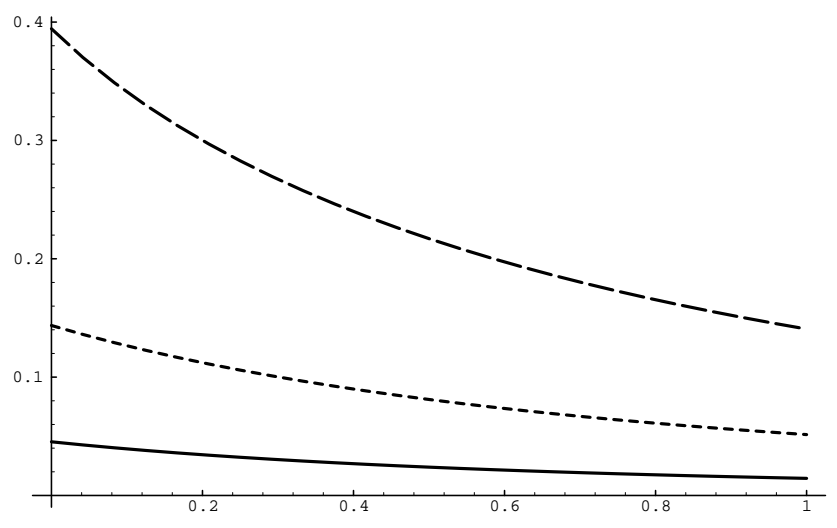

Figure 6: Price informativeness as a function of the transaction cost when informed traders are rational. The number of informed traders is set to 4 (solid line), 8 (short-dashed line) and 14 (long-dashed line); $\rho=.25, \kappa=\sigma_{x}=\sigma_{\varepsilon}=1$.

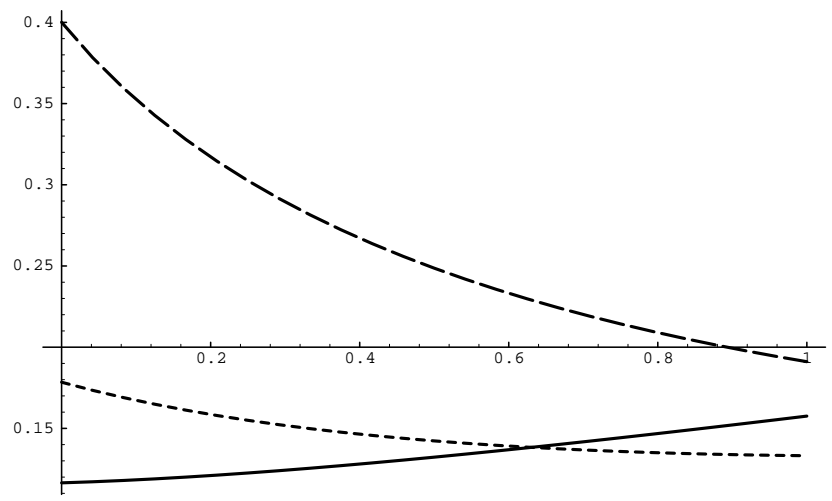

Figure 7: Price variance as a function of the transaction cost when informed traders are rational. The number of informed traders is set to 4 (solid line), 8 (short-dashed line) and 14 (long-dashed line); $\rho=.25, \kappa=\sigma_{x}=\sigma_{\varepsilon}=1$. 


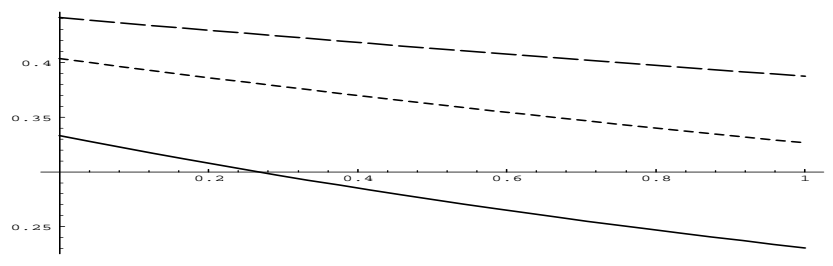

Figure 8: Liquidity trader's surplus as a function of the transaction cost when informed traders are naive. The number of informed traders is set to 4 (solid line), 8 (short-dashed line) and 14 (long-dashed line); $\rho=.25, \kappa=\sigma_{x}=\sigma_{\varepsilon}=1$.

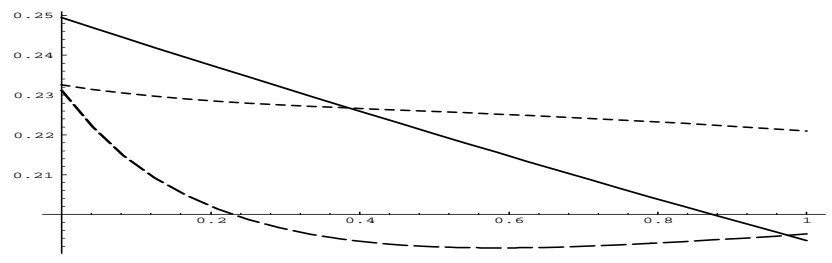

Figure 9: Liquidity trader's surplus as a function of the transaction cost when informed traders are rational. The number of informed traders is set to 4 (solid line), 8 (short-dashed line) and 14 (long-dashed line); $\rho=.25, \kappa=\sigma_{x}=\sigma_{\varepsilon}=1$.

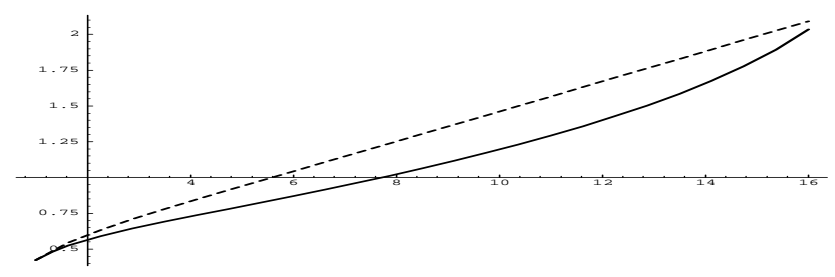

Figure 10: Expected trading volume as a function of the number of informed traders when they are naive (dashed line) and when they are rational (solid line); $\rho=.25, \kappa=\sigma_{x}=\sigma_{\varepsilon}=1$. 


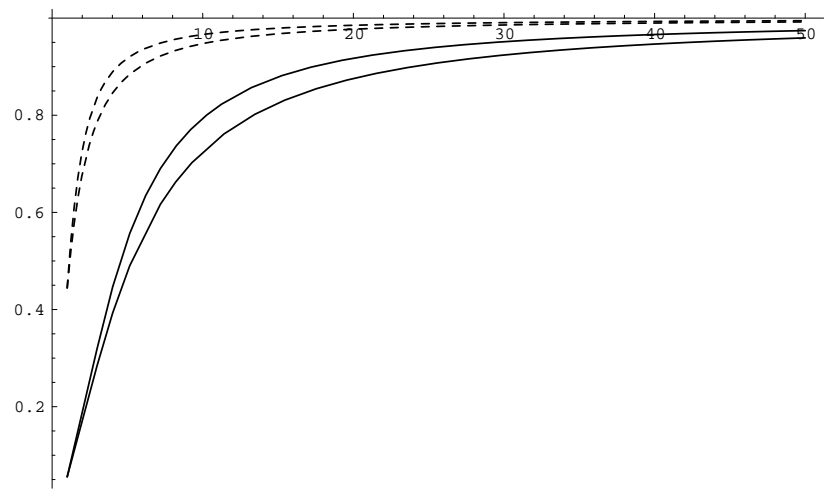

Figure 11: Price informativeness as a function of the number of informed traders for $\sigma_{\eta}=1$ (solid lines) and $\sigma_{\eta}=0.5$ (dashed lines) when they are naive (upper lines) and when they are rational (lower lines); $\sigma_{u}=0, \sigma_{x}=\sigma_{\varepsilon}=1, \kappa=4$.

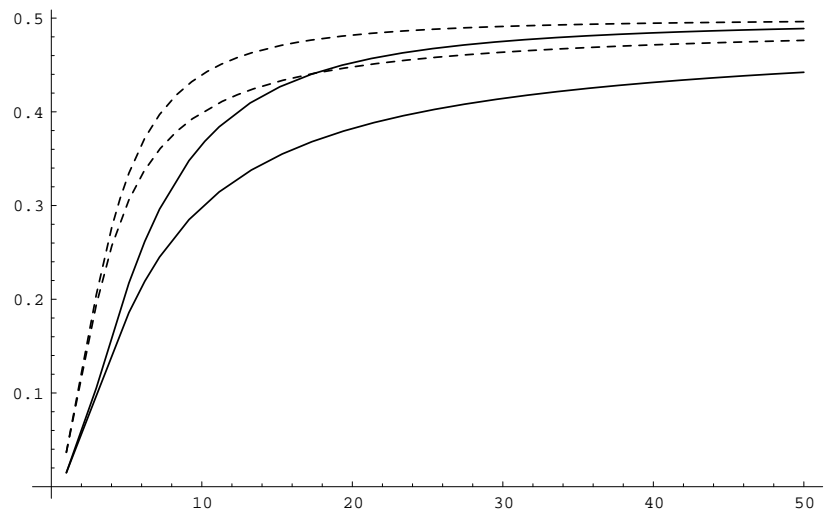

Figure 12: Price informativeness as a function of the number of informed traders for $\sigma_{\eta}=1$ (solid lines) and $\sigma_{\eta}=0.5$ (dashed lines) when they are naive (upper lines) and when they are rational (lower lines); $\sigma_{u}=1, \sigma_{x}=\sigma_{\varepsilon}=1, \kappa=4$. 


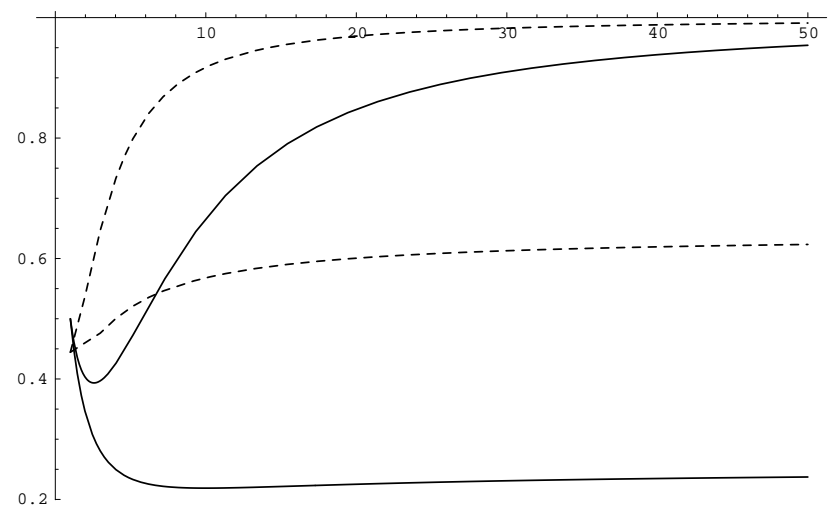

Figure 13: Price variance as a function of the number of informed traders for $\sigma_{\eta}=1$ (solid lines) and $\sigma_{\eta}=0.5$ (dashed lines) when they are naive (lower lines) and when they are rational (upper lines); $\sigma_{u}=0, \sigma_{x}=\sigma_{\varepsilon}=1, \kappa=4$.

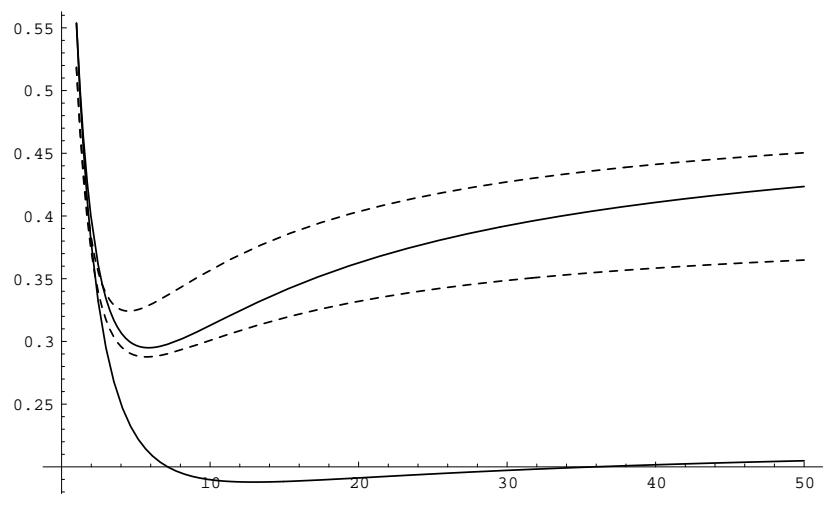

Figure 14: Price variance as a function of the number of informed traders for $\sigma_{\eta}=1$ (solid lines) and $\sigma_{\eta}=0.5$ (dashed lines) when they are naive (lower lines) and when they are rational (upper lines); $\sigma_{u}=1, \sigma_{x}=\sigma_{\varepsilon}=1, \kappa=4$. 